\title{
HAUSDORFF DIMENSION OF UNIFORMLY NON FLAT SETS WITH TOPOLOGY
}

\author{
GuY DAvid
}

Abstract

Let $d$ be an integer, and let $E$ be a nonempty closed subset of $\mathbb{R}^{n}$ Assume that $E$ is locally uniformly non flat, in the sense that for $x \in E$ and $r>0$ small, $E \cap B(x, r)$ never stays $\varepsilon_{0} r$-close to an affine $d$-plane. Also suppose that $E$ satisfies locally uniformly some appropriate $d$-dimensional topological nondegeneracy condition, like Semmes' Condition B. Then the Hausdorff dimension of $E$ is strictly larger than $d$. We see this as an application of uniform rectifiability results on Almgren quasiminimal (restricted) sets.

\section{Introduction}

Let $E$ be a closed subset in Euclidean space $\mathbb{R}^{n}$, and let an integer dimension $d<n$ be given. Suppose that at all scales and locations, $E$ is both uniformly non flat (relative to that dimension) and satisfies some $d$-dimensional topological nondegeneracy condition. We want to show that the Hausdorff dimension of $E$ is (strictly) larger than $d$.

A simple instance of this is when $d=1, E$ is connected, and its P. Jones numbers are such that $\beta_{E}(x, r) \geq \varepsilon$ for $x \in E$ and $0<r \leq 1$. Then C. J. Bishop and P. W. Jones [BJ2] showed that $E$ has Hausdorff dimension at least $d^{*}$, where $d^{*}>1$ depends only on $n$ and $\varepsilon$.

Here we shall keep the same condition to measure the uniform nonflatness of $E$. Call $\mathcal{P}$ the set of affine planes of dimension $d$ and set

$$
\beta_{E}(x, r)=\inf _{P \in \mathcal{P}}\left\{\sup \left\{r^{-1} \operatorname{dist}(y, P) ; y \in E \cap B(x, r)\right\}\right\}
$$

for $x \in \mathbb{R}^{n}$ and $r>0$. We require the existence of $\varepsilon_{0}>0$ and $r_{0}>0$ such that

$$
\beta_{E}(x, r) \geq \varepsilon_{0} \text { for all } x \in E \text { and } 0<r \leq r_{0} .
$$

2000 Mathematics Subject Classification. 49Q20, $28 \mathrm{~A} 75$.

Key words. Hausdorff dimension, quasiminimal sets, restricted sets, flatness. 
Note that using $L^{p}$ variants of $\beta_{E}(x, r)$ would only make (1.2) harder to check, and would thus lead to weaker theorems below. We could also prove the results below with a bilateral version of $\beta_{E}(x, r)$. That is, we could replace (1.2) with the weaker requirement that for every choice of $x \in E$ and $0<r \leq r_{0}$, we cannot find a plane $P$ such that $\operatorname{dist}(y, P) \leq \varepsilon_{0} r$ for every $y \in E \cap B(x, r)$ and $\operatorname{dist}(z, E) \leq \varepsilon_{0} r$ for every $z \in P \cap B(x, r)$. The proof would be the same (just replace the weak geometric lemma by its bilateral version near (6.10)). But this remark is essentially useless, because with our topological nondegeneracy condition, the weaker analogue of (1.2) implies (1.2) (with a smaller $\varepsilon_{0}$ ).

For the topological nondegeneracy condition, there seems to be no obvious best choice, so we shall present two main ones and a few consequences, and hope that they will turn out to be the right ones. The general idea, as in $[\mathbf{S e 2}]$, [DS2], and Section 12 of [DS3], is to require some property of topological nature (i.e., for instance, invariant under deformations) that would automatically imply that $E$ is at least $d$-dimensional. Connectedness (in dimension $d=1$ ), as in the result of Bishop and Jones, would be an example. We want to require something like this at all scales and locations, too.

For our first topological condition, we shall consider deformations of $E$ in balls $B(x, r)$. These deformations will be given by one-parameter families of Lipschitz mappings $\left\{\varphi_{t}\right\}, 0 \leq t \leq 1$, defined on $\mathbb{R}^{n}$, with the following properties:

$$
\varphi_{t}(\bar{B}(x, r)) \subset \bar{B}(x, r) \text { for each } t \in[0,1] ;
$$

for each $y \in \mathbb{R}^{n}, \varphi_{t}(y)$ is a continuous function of $t \in[0,1]$;

$$
\begin{gathered}
\varphi_{t}(y)=y \text { for } t=0 \text { and for } y \in \mathbb{R}^{n} \backslash B(x, r) ; \\
\operatorname{dist}\left(\varphi_{t}(y), E\right) \leq \alpha_{0} r \text { for } t \in[0,1] \text { and } y \in E \cap B(x, r),
\end{gathered}
$$

where the constant $\alpha_{0} \in(0,1)$ is given in advance. Thus we only allow ourselves to move $E \cap B(x, r)$ in a thin tube around $E$. This is important, because it may force $\varphi_{1}(E)$ to keep some of the topological properties of $E$.

Minor modifications of this are possible. For instance, we may require that $t \rightarrow \varphi_{t}$ be a continuous function of $t$, valued in Lipschitz functions. We could also have decided to define $\varphi_{t}$ only on $[E \cap B(x, r)] \cup \partial B(x, r)$, but it would then have been easy to extend it to $\mathbb{R}^{n}$. Note that in (1.3) we do not require any bound on the Lipschitz constant of $\varphi_{t}$, and probably we could even replace the Lipschitz requirement with simple continuity. 
For our first topological condition, we shall require the existence of two small positive constants $\eta_{0}$ and $\delta_{0}$ such that

$$
H^{d}\left[B\left(x,\left(1-\eta_{0}\right) r\right) \cap \varphi_{1}(E)\right] \geq \delta_{0} r^{d}
$$

when (1.3)-(1.6) hold. Let us already give the corresponding statement.

Theorem 1.8. For each choice of positive constants $\varepsilon_{0}, r_{0}, \alpha_{0}, \eta_{0}$, and $\delta_{0}$, we can find $d^{*}>d$ such that $\mathcal{H} \operatorname{dim}(E) \geq d^{*}$ whenever $E$ is a nonempty closed set in $\mathbb{R}^{n}$ such that (1.2) holds and

(1.9) (1.7) holds for all choices of $x \in E, 0<r \leq r_{0}$, and of Lipschitz deformations $\left\{\varphi_{t}\right\}$ with the properties (1.3), (1.4), (1.5), and (1.6).

Here $\mathcal{H} \operatorname{dim}(E)$ denotes the Hausdorff dimension of $E$. Thus our conclusion is that the Hausdorff measure $H^{m}(E)$ is infinite for every $m<d^{*}$. See for instance $[\mathbf{F a}]$ for details about $\mathcal{H} \operatorname{dim}$ and $H^{d}$.

We shall give in Section 2 a few additional comments about our topological condition (1.7), and in particular check that it is implied by S. Semmes' Condition B (a uniform separation property in codimension 1). However it is a little unpleasant that in (1.7) we require a lower bound on $H^{d}\left[B\left(x,\left(1-\eta_{0}\right) r\right) \cap \varphi_{1}(E)\right]$, rather than the more natural $H^{d}\left(\varphi_{1}(E \cap B(x, r))\right)$. Since we fear that the difference may cause trouble in potential applications, we shall give a slightly more general, but also somewhat more technical, version of Theorem 1.8, where we use approximations of $E$ by small $d$-dimensional skeletons. See Theorem 3.8 below.

Both theorems are easy to localize. That is, if $E$ satisfies the hypotheses of these theorems for all balls $B(x, r)$ contained in an open set $\Omega$, then $E \cap B(y, \rho)$ also has Hausdorff dimension at least $d^{*}$ when $y \in E \cap \Omega$ and $\rho>0$. This will be clear from the proof.

Our proof will be a little indirect; the general idea is the following. We shall proceed by contradiction, assume that $E$ is not too large, and construct a functional and a minimizer $F$ for this functional. By definition of the functional and the topological condition, $F$ will have a big intersection with a small modification $E_{\rho}$ of $E$. We shall also check that $F$ is a quasiminimal set (in the terminology of F. J. Almgren $[\mathbf{A l}]$, a restricted set), then use the local uniform rectifiability estimates from [DS3] to show that $F$ is very flat in most balls; a contradiction with (1.2) will ensue. All this will be in the spirit of Section 12 of [DS3]. See the beginning of Section 4 for a slightly more precise description of the argument.

Even though the author likes the indirect argument below, he is aware that there could be much simpler, direct proofs of Theorems 1.8 and 3.8. 
Potential applications for the results of this paper could be lower bounds on the dimension of limit sets of some groups, as was the case with the original paper of Bishop and Jones. But the author knows very little about the subject.

The author wishes to thank Leonid Potyagailo, who asked the question and told him about the potential applications to limit sets, A. Ancona for help with the topology in Section 2, and P. W. Jones for interesting discussions.

\section{Condition B and other topological nondegeneracy conditions}

The following condition was introduced by S. Semmes [Se1], in connection with $L^{2}$-boundedness of singular integral operators on subsets of $\mathbb{R}^{n}$. This is a topological condition of codimension 1 .

Definition 2.1. Let $E$ be a closed set in $\mathbb{R}^{n}$. We say that $E$ satisfies Condition B locally if there are constants $\alpha_{1}>0$ and $r_{0}>0$ such that, for all choices of $x \in E$ and $0<r \leq r_{0}$, we can find two balls $B_{1}$ and $B_{2}$ contained in $B(x, r) \backslash E$, of radius $\alpha_{1} r$, and such that $B_{1}$ and $B_{2}$ lie in different connected components of $B(x, r) \backslash E$.

We added "locally" because in [Se1] and related works, the property is assumed to hold for all $r<\operatorname{diam}(E)$. Also, we weakened the property a little, because normally one requires the balls $B_{1}$ and $B_{2}$ to be contained in different connected components of $\mathbb{R}^{n} \backslash E$.

Lemma 2.2. If the nonempty closed set E satisfies Condition B locally, then it satisfies the hypothesis (1.9) in Theorem 1.8, with $d=n-1$. Hence, if $E$ also satisfies (1.2) with $d=n-1$, then $\mathcal{H} \operatorname{dim}(E) \geq d^{*}>$ $n-1$, for some $d^{*}$ that depends only on $n, d, \alpha_{1}$, and $\varepsilon_{0}$.

So let $E$ satisfy Condition B locally, and let us prove (1.9); the rest of the lemma will follow from Theorem 1.8. We shall keep the same $r_{0}$ and take

$$
\alpha_{0}=\eta_{0}=\alpha_{1} / 2 \text { and } \delta_{0}=2^{-n+1} c_{n-1} \alpha_{1}^{n-1},
$$

where $c_{n-1}$ denotes the Lebesgue measure of the unit ball in $\mathbb{R}^{n-1}$.

Let $x \in E$ and $0<r \leq r_{0}$ be given, and let us apply Condition B. We get two balls $B_{1}=B\left(x_{1}, \alpha_{1} r\right)$ and $B_{2}=B\left(x_{2}, \alpha_{1} r\right)$, with the properties stated in Definition 2.1. Set $B_{j}^{\prime}=B\left(x_{j}, \alpha_{1} r / 2\right)$ for $j=1,2$.

Next let $\left\{\varphi_{t}\right\}$ be a family of Lipschitz functions, with the properties (1.3)-(1.6). Note that for $y \in E$ and $0 \leq t \leq 1,\left(\varphi_{t}(y), E\right) \leq \alpha_{0} r=$ 
$\alpha_{1} r / 2$, by (1.6) or (1.5). Thus

$$
\varphi_{t}(E) \text { never meets } B_{1}^{\prime} \cup B_{2}^{\prime} \text {. }
$$

Note that $\varphi_{t}(\partial B(x, r))=\partial B(x, r)$ does not meet $B_{1}^{\prime} \cup B_{2}^{\prime}$ either, by (1.5) and because $B_{j} \subset B(x, r)$. We claim that

(2.5) $\quad B_{1}^{\prime}$ and $B_{2}^{\prime}$ lie in different connected components of

$$
\mathbb{R}^{n} \backslash \varphi_{1}(E \cup \partial B(x, r)) .
$$

This comes directly from 4.3 on Chapter XVII of [Du, p. 360], (for instance). The point is that if $y_{1} \in B_{1}^{\prime}$ and $y_{2} \in B_{2}^{\prime}$, then $y_{1}$ and $y_{2}$ lie in different components of $\mathbb{R}^{n} \backslash[E \cup \partial B(x, r)]$ by definition of $B_{1}$ and $B_{2}$, and this stays the same with $\varphi_{t}(E)$ for all $t$, because $\varphi_{t}(E)$ never passes through $y_{1}$ or $y_{2}$. The proof uses Borsuk's theorem on extensions of maps into spheres, and Brouwer's theorem that says that the identity map on the sphere is not nullhomotopic.

Call $L$ the line through the centers $x_{1}$ and $x_{2}$, and $\pi$ the orthogonal projection onto the hyperplane $P$ through $x_{1}$ and perpendicular to $L$. Let us check that

$$
\pi\left[\varphi_{1}(E) \cap B\left(x, r-\eta_{0} r\right)\right] \text { contains } P \cap B_{1}^{\prime} .
$$

Let $\xi \in P \cap B_{1}^{\prime}$ be given, and call $L_{\xi}$ the line through $\xi$ parallel to $L$. Then $L_{\xi}$ meets $B_{1}^{\prime}$ and $B_{2}^{\prime}$, because $\pi\left(B_{1}^{\prime}\right)=\pi\left(B_{2}^{\prime}\right)=P \cap B_{1}^{\prime}$. Since $B_{1}^{\prime}$ and $B_{2}^{\prime}$ lie in different components of $\mathbb{R}^{n} \backslash \varphi_{1}(E \cup \partial B(x, r))$, we can find a point $z_{\xi}$ in $\varphi_{1}(E \cup \partial B(x, r)) \cap L_{\xi}$, somewhere between the intersections of $L_{\xi}$ with $B_{1}^{\prime}$ and $B_{2}^{\prime}$. Note that $z_{\xi}$ lies in the convex hull of $B_{1}^{\prime} \cup B_{2}^{\prime}$, which itself is contained in $B\left(x, r-\alpha_{1} r / 2\right)=B\left(x, r-\eta_{0} r\right)$. So $z_{\xi} \in$ $\varphi_{1}(E) \cap B\left(x, r-\eta_{0} r\right)$, and since $\pi\left(z_{\xi}\right)=\xi$, we see that $\xi \in \pi\left[\varphi_{1}(E) \cap\right.$ $\left.B\left(x, r-\eta_{0} r\right)\right]$, as needed for (2.6).

Since $\pi$ is Lipschitz with constant $1,(2.6)$ says that

$$
\begin{aligned}
H^{d}\left(\varphi_{1}(E) \cap B\left(x, r-\eta_{0} r\right)\right) & \geq H^{d}\left(\pi\left[\varphi_{1}(E) \cap B\left(x, r-\eta_{0} r\right)\right]\right) \\
& \geq H^{d}\left(P \cap B\left(x_{1}, \alpha_{1} r / 2\right)\right) \\
& =2^{-n+1} c_{n-1} \alpha_{1}^{n-1} r^{d}
\end{aligned}
$$

which is exactly (1.7) with $\delta_{0}=2^{-n+1} c_{n-1} \alpha_{1}^{n-1}$.

This completes our proof of (1.9), and Lemma 2.2 follows.

It is reasonably easy to come up with generalizations of Condition B in higher codimensions, but it is a little harder to guess which one is the most natural. We shall give such a condition here, more as an example of what can be done than a final answer to a natural problem. The following definition is a local version of Definition 3 on [Da, p. 106]. 
Definition 2.8. Let $E$ be a closed set in $\mathbb{R}^{n}$. We say that $E \in S S(d, n)$ locally if there are constants $\alpha_{1}>0$ and $r_{0}>0$ such that, for all choices of $x \in E$ and $0<r \leq r_{0}$, we can find an affine subspace $W$ of dimension $n-d$ and an Euclidean sphere $\Sigma \subset W \cap B(x, r / 2)$ of dimension $n-d-1$, such that

$$
\operatorname{dist}(\Sigma, E) \geq 2 \alpha_{1} r
$$

and $\Sigma$ is linked with $E$ in $B(x, r)$, in the sense that there is no oneparameter family $\left\{f_{t}\right\}$ of continuous functions on $\mathbb{R}^{n}$ such that

$$
f_{t}(\bar{B}(x, r)) \subset \bar{B}(x, r) \text { for each } t \in[0,1],
$$

for each $y \in \mathbb{R}^{n}, f_{t}(y)$ is a continuous function of $t \in[0,1]$,

$$
\begin{gathered}
f_{t}(y)=y \text { for } t=0 \text { and for } y \in \mathbb{R}^{n} \backslash B(x, r), \\
\operatorname{dist}\left(f_{t}(y), \Sigma\right) \geq \alpha_{1} r \text { for } t \in[0,1] \text { and } y \in E \cap B(x, r),
\end{gathered}
$$

and

$$
f_{1}(E \cap B(x, r)) \subset \partial B(x, r) .
$$

In other words, there is no way to deform $E \cap B(x, r)$ inside of $\bar{B}(x, r)$ so that it lands on $\partial B(x, r)$, without crossing a small neighborhood of $\Sigma$.

When $d=n-1, \Sigma$ is composed of two points, and our condition looks a lot like Condition B, except that instead of saying that the two centers of the $B_{j}$ in Condition B lie in different components of $\mathbb{R}^{n} \backslash E$, we say that we cannot deform $E$ past the two points without crossing them. But here the conditions are equivalent, by Borsuk's and Brouwer's theorems.

In higher codimensions, one could come up with other ways to say that $E$ is linked with $\Sigma$, and it is not clear that they are all equivalent. The linking condition in Definition 2.8 is probably not the most natural one (as the case of codimension 1 suggests), but at least we can use it easily. Also, there are probably a few algebraic ways to make sure that $E$ and $\Sigma$ are linked and $E \in S S(d, n)$ locally, but this is a slightly different subject. See Remark 2.24 for a little more about this.

Lemma 2.15. If $E$ is a closed set and $E \in S S(d, n)$ locally, then we can find $\alpha_{0}, \delta_{0}$, and $\eta_{0}$ such that (1.7) holds for all choices of $x \in E$, $0<r \leq r_{0}$, and all families $\left\{\varphi_{t}\right\}$ of Lipschitz deformations such that (1.3), (1.4), (1.5), and (1.6) hold. 
In other words, (1.9) holds. The constants $\alpha_{0}, \delta_{0}$, and $\eta_{0}$ depend only on the constants in $S S(d, n)$; in fact, we can keep the same $r_{0}$ and take

$$
\alpha_{0}=\alpha_{1}, \eta_{0}=1 / 2, \text { and } \delta_{0}=2^{-d} c_{d} \alpha_{1}^{d} .
$$

Now Theorem 2.9 says that if $E$ is closed, nonempty, and $E \in S S(d, n)$ and (1.2) holds, then the Hausdorff dimension of $E$ is at least $d^{*}$, where $d^{*}>d$ depends only on $n, d, \alpha_{1}$, and $\varepsilon_{0}$.

Let us prove the lemma. Choose constants as in (2.16). As before, we start with $x \in E$ and $0<r \leq r_{0}$, Definition 2.8 gives a sphere $\Sigma$, and we want to use it to prove (1.7) for all families $\left\{\varphi_{t}\right\}$ of Lipschitz functions that satisfy $(1.3)-(1.6)$.

So we give ourselves such a family, assume that (1.7) fails, and try to reach a contradiction. The idea will be to use $\left\{\varphi_{t}\right\}$ to find deformations $\left\{f_{t}\right\}$ that satisfy $(2.10)-(2.14)$.

Note that $\left\{\varphi_{t}\right\}$ automatically satisfies (2.10)-(2.12), by (1.3)-(1.5). Also,

$$
\operatorname{dist}\left(\varphi_{t}(E), \Sigma\right) \geq \operatorname{dist}(E, \Sigma)-\alpha_{0} r \geq \alpha_{0} r
$$

by (1.6), (2.9), and because $\alpha_{0}=\alpha_{1}$. In particular, (2.13) holds for $\left\{\varphi_{t}\right\}$. But $\varphi_{1}$ does not necessarily satisfy (2.14); we only know that (1.7) fails, i.e., that

$$
H^{d}\left[\varphi_{1}(E) \cap B(x, r / 2)\right]<\delta_{0} r^{d}=2^{-d} c_{d} \alpha_{1}^{d} r^{d},
$$

by (2.16). We have to deform $\varphi_{1}(E)$ some more, to send it to $\partial B(x, r)$.

Call $x_{0}$ the center of $\Sigma, W^{\perp}$ the affine $d$-plane through $x_{0}$ perpendicular to $W$, and $\pi$ the orthogonal projection onto $W^{\perp}$. Then (2.18) says that

$$
H^{d}\left[\varphi_{1}(E) \cap B(x, r / 2)\right]<H^{d}\left(W^{\perp} \cap B\left(x_{0}, \alpha_{1} r / 2\right)\right) .
$$

Hence $\pi\left(\varphi_{1}(E) \cap B(x, r / 2)\right)$ does not contain $W^{\perp} \cap B\left(x_{0}, \alpha_{1} r / 2\right)$.

Let $z \in W^{\perp} \cap B\left(x_{0}, \alpha_{1} r / 2\right)$ be such that $D=\pi^{-1}(z) \cap B(x, r / 2)$ does not meet $\varphi_{1}(E)$. Set $U=\left\{y \in B(x, r)\right.$; $\left.\operatorname{dist}(y, \Sigma)<\alpha_{1} r\right\}$, and call $V$ the convex hull of $U$. Thus $V$ is an $\alpha_{1} r$-neighborhood of the ball of $W$ enclosed in $\Sigma$.

Also set $D_{\varepsilon}=\{y \in V$; dist $(y, D)<\varepsilon\}$. Since $\varphi_{1}(E)$ is closed and does not meet $D$, we can choose $\varepsilon<\alpha_{1} r / 2$ so small that $D_{\varepsilon}$ does not meet $\varphi_{1}(E)$. 
Now we can find a continuous deformation $\left\{\psi_{t}\right\}, 0 \leq t \leq 1$, such that $\psi_{0}(y)=y$ everywhere, $\psi_{t}(y)=y$ when $y \in \mathbb{R}^{n} \backslash V$,

$$
\psi_{t}\left(\bar{V} \backslash\left[U \cup D_{\varepsilon}\right]\right) \subset \bar{V} \backslash\left[U \cup D_{\varepsilon}\right] \text { for all } t,
$$

and

$$
\psi_{1}\left(V \backslash\left[U \cup D_{\varepsilon}\right]\right) \subset \partial V .
$$

Let us just give an idea of the construction. We first define $\psi_{1}$, and then we shall take $\psi_{t}(y)=t y+(1-t) \psi_{1}(y)$. We keep $\psi_{1}(y)=y$ on $D$. For $y \in V \backslash D_{\varepsilon}$, we call $y^{*}$ the point of $D$ such that $y^{*}-y$ is parallel to $W^{\perp}$, and then let $\psi_{1}(y)$ be the point of the half line from $y^{*}$ through $y$ that lies in $\partial V$. In other words, we move $y$ straight away from $D$ and parallel to $W^{\perp}$ until we hit $\partial V$. For the rest of $V$, i.e., on $V \cap D_{\varepsilon}$, we interpolate nicely between the two definitions. The properties (2.20) and (2.21) are easy to check; the main point for (2.20) is that if $y \in V \backslash\left[U \cup D_{\varepsilon}\right]$ then all the points of the half-line from $y^{*}$ through $y$ that lie after $y$ also lie out of $U \cup D_{\varepsilon}$.

We complete our deformation $\left\{\psi_{t}\right\}$ by another continuous family $\left\{h_{t}\right\}$, $0 \leq t \leq 1$, such that $h_{t}(y)=y$ for $t=0$ and for $y \in \mathbb{R}^{n} \backslash B(x, r)$, $h_{t}(\bar{B}(x, r)) \subset \bar{B}(x, r)$ for all $t$,

$$
\begin{aligned}
h_{t}(B(x, r) \backslash V) \subset \bar{B}(x, r) \backslash V & \text { for all } t, \\
& \text { and } h_{1}(\bar{B}(x, r) \backslash V) \subset \partial B(x, r) .
\end{aligned}
$$

This one is very easy to construct, since $V$ is essentially a smaller ball inside $B(x, r)$; we just need to push the points out to $\partial B(x, r)$.

Our family $\left\{f_{t}\right\}$ is obtained by composing the three families above. That is, we set $f_{t}(y)=\varphi_{3 t}(y)$ for $0 \leq t \leq 1 / 3$, then $f_{t}(y)=\psi_{3 t-1}\left(\varphi_{1}(y)\right)$ for $1 / 3<t \leq 2 / 3$, and finally $f_{t}(y)=h_{3 t-2}\left(\psi_{1}\left(\varphi_{1}(y)\right)\right)$ for $t>2 / 3$. The properties (2.10)-(2.12) are straightforward. Let us check (2.13).

When $t \leq 1 / 3,(2.13)$ follows from $(2.17)$. We also get that $\varphi_{1}(E) \subset$ $\mathbb{R}^{n} \backslash\left[U \cup D_{\varepsilon}\right]$, by definition of $U$ and $D_{\varepsilon}$. Then (2.20) says that $f_{t}(E) \subset$ $\mathbb{R}^{n} \backslash\left[U \cup D_{\varepsilon}\right]$ for $1 / 3<t \leq 2 / 3$, which proves (2.13) in that case. Also note that

$$
f_{2 / 3}(E)=\psi_{1}\left(\varphi_{1}(E)\right) \subset \mathbb{R}^{n} \backslash V,
$$

by (2.21). Finally, for $t>2 / 3, f_{t}(E)=h_{3 t-2}\left(\psi_{1}\left(\varphi_{1}(E)\right)\right)$ stays out of $V$ by (2.22), and hence (2.13) holds.

Our last condition (2.14) holds because $f_{1}(E \cap B(x, r))=h_{1}\left(\psi_{1}\left(\varphi_{1}(E \cap\right.\right.$ $B(x, r))) \subset h_{1}(\bar{B}(x, r) \backslash V) \subset \partial B(x, r)$, by (2.23) and (2.22).

By Definition 2.8, such a family $\left\{f_{t}\right\}$ cannot exist; this gives the desired contradiction and proves Lemma 2.15. 
Remark 2.24. It would be easy to prove the analogue of Lemma 2.15 with a somewhat weaker definition of $S S(d, n)$. First, the Euclidean sphere $\Sigma$ could be replaced with a bilipschitz image of such a sphere, for instance. In $[\mathbf{D a}]$ this was hard to do because one wanted to know that $E$ has "big projection", and for this one really wanted to show that the straight projection $\pi(E \cap B(x, r / 2))$ contains $W^{\perp} \cap B\left(x_{0}, \alpha_{1} r / 2\right)$. The argument was the same as above (for $\varphi_{t}(y) \equiv y$ ); see below (2.19). Here we just want to prove (1.7) (or contradict (2.18)), and for this a bilipschitz image of a sphere is enough. Since the point is merely to get (1.7), one could probably replace spheres by other objects too.

In fact, we could get (1.7) from various other linking conditions, like the existence of a homotopically nontrivial mapping from $E \cap B(x, r)$ to a sphere $\mathbb{S}^{d}$. We do not want to try to give a list here, because we would surely miss the most relevant example. But at least we should say that (1.7) has a stability property that should makes it pleasant to check. That is, if we choose $\delta_{0}$ small enough, depending on $n, d$, and $\alpha_{0}$, then either (1.7) holds (for all deformations $\left\{\varphi_{t}\right\}$ in $B(x, t)$ ), or else we can even find $\left\{\varphi_{t}\right\}$ such that $B\left(x,\left(1-\eta_{0}\right) r\right) \cap \varphi_{1}(E)$ has Hausdorff dimension at most $d-1$. We can get this by a projection on skeletons, a little bit like when we will construct $f$ and $\pi$ in Section 5 . The reader in kindly invited to consult Subsection 12.2 of [DS3] (called Stability of Sets) for this; more ideas on topological conditions that may lead to (1.7) can be found in Subsection 12.3 of [DS3] (Topological Interpretations).

\section{Our main technical topological condition}

For our main technical topological nondegeneracy condition, we need to introduce dyadic cubes and skeletons. Let $\rho>0$ be a small number; the reader may think of $\rho$ as a small power of 2 , but since we often work at one scale at a time, this does not really matter. Our set of dyadic cubes of size $\rho$ is

$$
\mathcal{Q}(\rho)=\left\{Q=\rho[0,1]^{n}+\rho \ell ; \ell \in \mathbb{Z}^{n}\right\} .
$$

For each $Q \in \mathcal{Q}(\rho)$ and each integer $m \in[0, n]$, we denote by $S_{m}(Q)$ the $m$-dimensional skeleton of $Q$. Thus $S_{0}(Q)$ is the set of all vertices of $Q$, $S_{n}(Q)$ is the cube $Q$ itself, and in general $S_{m}(Q)$ is the union of all the $m$-dimensional faces of $Q$. It will be convenient to replace the closed set $E$ with

$$
E_{\rho}=\bigcup_{Q \in \mathcal{Q}(\rho) ; Q \cap E \neq \emptyset} S_{d}(Q),
$$


where $d$ is the same fixed integer as in the introduction. One of the points is that $E_{\rho}$ has locally finite $H^{d}$-measure, while we expect $E$ to be much larger. And the difference is not so large, because we can deform $E$ onto a subset of $E_{\rho}$, at least if $\mathcal{H} \operatorname{dim}(E)<d+1$. [See Lemma 3.14.]

Our second topological nondegeneracy condition TND is the following. We assume that for each choice of a big constant $C_{1}>0$, there are (small) positive constants $r_{0}, \alpha<1, \eta$, and $\delta_{1}$ such for every choice of

$$
x_{1} \in E, 0<r_{1} \leq r_{0}, \text { and } 0<\rho \leq \frac{\alpha r_{1}}{2 \sqrt{n}}
$$

(the reader should not pay too much attention to the constant $2 \sqrt{n}$ here, the point is merely to make sure that points of $E_{\rho}$ lie within $\frac{\alpha r}{2}$ of $E$ ) such that

$$
H^{d}\left(E_{\rho} \cap B\left(x_{1}, r_{1}\right)\right) \leq C_{1} r_{1}^{d},
$$

there is a ball $B(x, r)$ centered on $E$ and contained in $B\left(x_{1}, r_{1}\right)$ such that, for each one-parameter family $\left\{\varphi_{t}\right\}_{0 \leq t \leq 1}$ of Lipschitz functions on $\mathbb{R}^{n}$ that satisfy (1.3), (1.4), (1.5), and for which

$$
\operatorname{dist}\left(\varphi_{t}(y), E\right) \leq \alpha r_{1} \text { for } t \in[0,1] \text { and } y \in E_{\rho} \cap B(x, r),
$$

we have that

$$
H^{d}\left(\varphi_{1}\left(E_{\rho} \cap B(x, r)\right)\right) \geq \delta_{1} r_{1}^{d}+H^{d}\left(E_{\rho} \cap A_{\eta r_{1}}(x, r)\right),
$$

where we set

$$
A_{\eta r_{1}}(x, r)=B(x, r) \backslash B\left(x, r-\eta r_{1}\right) .
$$

When this condition is satisfied, we shall also say that $E \in T N D$.

We still have an unpleasant boundary term in (3.6) (namely, $H^{d}\left(E_{\rho} \cap\right.$ $\left.\left.A_{\eta r_{1}}(x, r)\right)\right)$, but hopefully it will be easier to control in applications than the one in Theorem 1.8, in particular because we get some limited control from the extra condition (3.4).

Also, we get some extra flexibility by allowing our nondegeneracy condition (3.6) to hold only on some balls $B(x, r)$.

Note that we did not specify officially that $r \geq C^{-1} r_{1}$, but since we can apply (3.6) with $\varphi_{t}(y) \equiv y$, we get that $E_{\rho} \cap B(x, r) \not \subset A_{\eta r_{1}}(x, r)$, hence $r \geq \eta r_{1}$. This is also why we put $\delta_{1} r_{1}^{d}$ (and not $\delta_{1} r^{d}$ ) in the right-hand side.

Incidentally, the statement below would still be true if we replaced the ball $B(x, r)$ with a cube, or some other object that is bilipschitzequivalent to a ball (and modified the annulus $A_{\eta r_{1}}(x, r)$ accordingly), but hopefully we shall not need to know this. 
Theorem 3.8. If the nonempty closed set $E \subset \mathbb{R}^{n}$ satisfies (1.2) (for some choice of $r_{0}>0$ and $\varepsilon_{0}>0$ ) and $T N D$, then $\mathcal{H} \operatorname{dim}(E) \geq d^{*}$ for some $d^{*}>d$.

As we shall see at the beginning of Section 7 , we shall only need to apply $T N D$ with one value of $C_{1}$, which depends only on $n$ and $d$, and then $d^{*}$ will depend only on $n, d, \varepsilon_{0}$, and the values of $\alpha, \eta$, and $\delta_{1}$ that we get by applying $T N D$ for this choice of $C_{1}$.

In the rest of this section, we want to show that Theorem 1.8 follows from Theorem 3.8. Hopefully, this will also convince the reader that $T N D$ is not too difficult to prove. The later sections will be devoted to the proof of Theorem 3.8.

So let $E$ satisfy the hypotheses of Theorem 1.8, and suppose in addition that

$$
\mathcal{H} \operatorname{dim}(E)<d+1
$$

(otherwise, there is nothing to prove). We want to show that $E \in T N D$, so we give ourselves a large constant $C_{1}$, and we try to find positive constants so that the condition in TND holds. For future reference, let us announce already that we shall keep the same value for $r_{0}$ and choose

$$
\alpha=\frac{1}{2} \operatorname{Min}\left(\eta_{0}, \alpha_{0}\right), \eta=2^{-d-1} 10^{-1} C_{1}^{-1} \delta_{0}, \text { and } \delta_{1}=2^{-d-1} \delta_{0} .
$$

Let $x_{1}, r_{1}$, and $\rho$ be as in (3.3), and assume that $H^{d}\left(E_{\rho} \cap B\left(x_{1}, r_{1}\right)\right) \leq$ $C_{1} r_{1}^{d}$, as in (3.4). We take $x=x_{1}$ and choose $r$ such that

$$
r_{1} / 2 \leq r \leq 2 r_{1} / 3
$$

and the mass of $E_{\rho}$ in the thin annulus $A_{\eta r_{1}}(x, r)=B(x, r) \backslash B\left(x, r-\eta r_{1}\right)$ is very small. More precisely, note that we can find more than $(10 \eta)^{-1}$ choices or $r$ such that (3.11) holds and the $A_{\eta r_{1}}(x, r)$ are disjoint, so we can pick $r$ such that

$$
\begin{aligned}
H^{d}\left(E_{\rho} \cap A_{\eta r_{1}}(x, r)\right) & \leq 10 \eta H^{d}\left(E_{\rho} \cap B\left(x_{1}, r_{1}\right)\right) \\
& \leq 10 \eta C_{1} r_{1}^{d} \leq 2^{-d-1} \delta_{0} r_{1}^{d} \leq \delta_{1} r_{1}^{d},
\end{aligned}
$$

by (3.4) and by our choice of $\eta$ and $\delta_{1}$ in (3.10).

So we picked $x \in E$ and $r$ such that $B(x, r) \subset B\left(x_{1}, r_{1}\right)$. What we need to do now is take a family $\left\{\varphi_{t}\right\}$ of Lipschitz functions on $\mathbb{R}^{n}$ that satisfies (1.3), (1.4), (1.5), and (3.5), and show that it satisfies (3.6). By (3.12), it will be enough to check that

$$
H^{d}\left(\varphi_{1}\left(E_{\rho} \cap B(x, r)\right)\right) \geq 2 \delta_{1} r_{1}^{d} .
$$


To do so, we want to use $\left\{\varphi_{t}\right\}$ to construct a deformation $\left\{f_{t}\right\}$ of $E$, and then apply the hypotheses of Theorem 1.8 to $\left\{f_{t}\right\}$. So it is a good idea to first deform $E$ into a subset of $E_{\rho}$.

Call $\mathcal{Q}_{1}(\rho)$ the set of cubes $Q \in \mathcal{Q}(\rho)$ such that $Q$ meets $B(x, r)$, $\mathcal{Q}_{2}(\rho)$ the set of cubes $Q \in \mathcal{Q}(\rho)$ that meet a cube of $\mathcal{Q}_{1}(\rho)$, and set $D_{j}=\bigcup_{Q \in \mathcal{Q}_{j}(\rho)} Q$ for $j=1,2$.

Lemma 3.14. There is a Lipschitz function $\psi: \mathbb{R}^{n} \rightarrow \mathbb{R}^{n}$ such that

$$
\begin{array}{ll}
\psi(x)=x & \text { for } x \in \mathbb{R}^{n} \backslash D_{2}, \\
\psi(Q) \subset Q & \text { for } Q \in \mathcal{Q}(\rho),
\end{array}
$$

and

$$
\psi(E) \cap Q \subset S_{d}(Q) \quad \text { for } Q \in \mathcal{Q}_{1}(\rho) .
$$

The construction of $\psi$ is a standard Federer-Flemming argument. We shall obtain $\psi$ as a composition of various projection mappings onto skeletons of smaller and smaller dimensions. Let us give a rapid proof here; the reader may also look at Proposition 3.1 in [DS3], where a variant of the lemma is proved with more details.

We start with a "projection" $\pi_{1}$ that will send $E \cap D_{2}$ into faces of dimension $n-1$. Let us first define $\pi_{1}$ on $Q$ when $Q$ is a cube of $\mathcal{Q}_{2}(\rho)$. Choose a point $c_{Q} \in \operatorname{int}(Q) \backslash E$ (which is possible by (3.9)), and decide already that the restriction of $\pi_{1}$ to $[Q \cap E] \cup \partial Q$ will be the radial projection on $\partial Q$ centered at $c_{Q}$. That is, $\pi_{1}(y)$ is the intersection of $\partial Q$ with the half line through $y$ that emanates from $c_{Q}$. Then extend $\pi_{1}$ to $Q$, so that $\pi_{1}(Q) \subset Q$ and $\pi_{1}$ is Lipschitz on $Q$. The Lipschitz constant may be enormous, if $c_{Q}$ is very close to $E$, but this does not matter. Notice that we get a coherent definition of $\pi_{1}$ on $D_{2}$. Indeed, if $Q$ and $Q^{\prime} \in \mathcal{Q}_{2}(\rho)$ meet, then both definitions yield $\pi_{1}(y)=y$ on $Q \cap Q^{\prime}$, because $Q \cap Q^{\prime}$ is contained in $\partial Q$ and in $\partial Q^{\prime}$. We take $\pi_{1}(y)=y$ out of $D_{2}$. This is still coherent with our definition of $\pi_{1}$ on $D_{2}$.

So we constructed a first Lipschitz function $\pi_{1}$ on $\mathbb{R}^{n}$ such that $\pi_{1}(Q) \subset$ $Q$ for $Q \in \mathcal{Q}(\rho)$ and for which $\pi_{1}(E)$ is contained in the union of $E \cap\left(\mathbb{R}^{n} \backslash\right.$ $\left.D_{2}\right)$ and of the $(n-1)$-dimensional faces of the cubes $Q, Q \in \mathcal{Q}_{2}(\rho)$. Note also that $\mathcal{H} \operatorname{dim}\left(\pi_{1}(E)\right)<d+1$, by (3.9) and because $\pi_{1}$ is Lipschitz.

If $d=n-1$, we can take $\psi=\pi_{1}$. Otherwise, we have to continue the construction. We want to send points of the $(n-1)$-dimensional faces of the cubes $Q, Q \in \mathcal{Q}_{1}(\rho)$, to the boundary of these faces. Notice that when we talk about the boundary $\partial F$ and the interior int $(F)$ of an 
$m$-dimensional face $F$, we shall really see $F$ as a $m$-dimensional object. Thus $\partial F$ is an union of faces of dimension $m-1$.

For each $(n-1)$-dimensional face $F$ contained in $D_{1}$, we choose a point $c_{F} \in \operatorname{int}(F) \backslash \pi_{1}(E)$. Such a point exists because $\mathcal{H} \operatorname{dim}\left(\pi_{1}(E)\right)<$ $d+1 \leq n-1$.

We define $\pi_{2}$ first on $\partial F \cup\left(\pi_{1}(E) \cap F\right)$, where we demand that $\pi_{2}(y)$ coincides with the radial projection on $\partial F$ with center $c_{F}$. Then we extend this to a Lipschitz function defined on $F$, and such that $\pi_{2}(F) \subset F$. We do this on each $(n-1)$-face $F$ contained in $D_{1}$, and this gives a definition of $\pi_{2}$ on $\bigcup_{\mathcal{Q}_{1}(\rho)} S_{n-1}(Q)$. This definition is coherent, because $\pi_{2}$ is the identity on the intersections of $(n-1)$-faces of cubes $Q \in \mathcal{Q}_{1}(\rho)$.

Then we define $\pi_{2}$ on the $(n-1)$-faces $F^{\prime}$ of cubes $Q \in \mathcal{Q}_{2}(\rho)$ such that $F^{\prime} \not \subset D_{1}$. We set $\pi_{2}(y)=y$ on $F^{\prime}$; notice that this is coherent with our definition on the faces $F \subset D_{1}$, because $F \cap F^{\prime} \subset \partial F$ for such a combination of faces. This gives a definition of $\pi_{2}$ on all the faces of dimension $n-1$ in $D_{2}$. We extend this to the whole $D_{2}$, making sure that $\pi_{2}(Q) \subset Q$ for $Q \in \mathcal{Q}_{2}(\rho)$ and $\pi_{2}(y)=y$ on $\partial D_{2}$. Then we set $\pi_{2}(y)=y$ out of $D_{2}$, and we get a Lipschitz map $\pi_{2}$ on $\mathbb{R}^{n}$.

Now $\pi_{2} \circ \pi_{1}(E)$ is contained in $\mathbb{R}^{n} \backslash D_{2}$, plus the interior of the hyperfaces $F$ of cubes $Q \in \mathcal{Q}_{2}(\rho)$ such that $F \not \subset D_{1}$ (i.e., that are not contained in a cube of $\left.\mathcal{Q}_{1}(\rho)\right)$, plus the faces of dimension $n-2$ of cubes $Q \in \mathcal{Q}_{1}(\rho)$. This comes from our earlier description of $\pi_{1}(E)$, and the fact the we moved all the points of $\pi_{1}(E)$ that lied on a $(n-1)$-face $F \subset D_{1}$ to a boundary $\partial F$. Also, $\mathcal{H} \operatorname{dim}\left(\pi_{2} \circ \pi_{1}(E)\right) \leq \mathcal{H} \operatorname{dim}\left(\pi_{1}(E)\right)<d+1$, because $\pi_{2}$ is Lipschiz.

If $d=n-2$, we can take $\psi=\pi_{2} \circ \pi_{1}$; see the short justification below. Otherwise, we still need to project. We look first at faces of dimension $n-2$ of cubes $Q \in \mathcal{Q}_{1}(\rho)$. For each such face $F$, we pick a point $c_{F} \in \operatorname{int}(F) \backslash \pi_{2} \circ \pi_{1}(E)$. Such a point exists, because $\mathcal{H} \operatorname{dim}\left(\pi_{2} \circ\right.$ $\left.\pi_{1}(E)\right)<d+1 \leq n-2$. We decide that on $\partial F \cup\left[\pi_{2} \circ \pi_{1}(E)\right], \pi_{3}$ coincides with the radial projection on $\partial F$ with center $c_{F}$. On the other faces of dimension $n-2$, we set $\pi_{3}(y)=y$ (which is coherent).

Then we extend $\pi_{3}$ to the faces of dimension $n-1$. When we do this, we make sure that $\pi_{3}(F) \subset F$ for each such face, that $\pi_{3}(y)=y$ on the faces that do not touch $D_{1}$, and also that the interior of $F$ is sent to the interior of $F$. Then we extend $\pi_{3}$ to $D_{2}$, still making sure that $\pi_{3}(Q) \subset Q$ for $Q \in \mathcal{Q}(\rho)$. Finally we set $\pi_{3}(y)=y$ out of $D_{2}$.

Now $\pi_{3} \circ \pi_{2} \circ \pi_{1}(E)$ is contained in $\mathbb{R}^{n} \backslash D_{2}$, plus the interior of the hyperfaces $F$ of cubes $Q \in \mathcal{Q}_{2}(\rho)$ such that $F \not \subset D_{1}$, plus the faces of dimension $n-3$ of cubes $Q \in \mathcal{Q}_{1}(\rho)$. Indeed, if $z \in \pi_{2} \circ \pi_{1}(E)$, then either 
$z \in \mathbb{R}^{n} \backslash D_{2}$ and $\pi_{3}(z)=z$, or $z$ lies in the interior of some hyperface $F$ of a cube $Q \in \mathcal{Q}_{2}(\rho)$, with $F \not \subset D_{1}$, and then $\pi_{3}(z)$ also lies in the interior of $F$, or else $z$ lies in a face of dimension $n-2$ of a cube $Q \in \mathcal{Q}_{1}(\rho)$, and then $\pi_{3}(z)$ lies on the boundary of that face.

We continue this procedure until we get to $d$-dimensional faces. Each time, we first construct $\pi_{k}$ on the faces $F$ of dimension $n-d+1$ of cubes $Q \in \mathcal{Q}_{1}(\rho)$. There we chose a center $c_{F} \in \operatorname{int}(F) \backslash \pi_{k-1} \circ \cdots \circ \pi_{1}(E)$, and decide that for $y \in \partial F \cup\left[\pi_{k-1} \circ \cdots \circ \pi_{1}(E) \cap F\right], \pi_{k}(y)$ is the radial projection on $\partial F$ of $y$ (with center $c_{F}$ ). We set $\pi_{k}(y)=y$ on the other faces of dimension $n-d+1$. Then we extend $\pi_{k}$ to faces $F^{\prime}$ of larger and larger dimensions, making sure that $\pi_{k}\left(F^{\prime}\right) \subset F^{\prime}$ for all $F^{\prime}$, and that $\pi_{3}(y)=y$ on the faces that do not touch $D_{1}$. When we get to hyperfaces, we also make sure that $\pi_{k}\left(\operatorname{int}\left(F^{\prime}\right)\right) \subset \operatorname{int}\left(F^{\prime}\right)$ for the hyperfaces $F^{\prime}$ that are contained in $D_{2}$, but not in $D_{1}$.

Eventually we get to $k=n-d$, and then $\pi_{k} \circ \cdots \circ \pi_{1}(E)$ is contained in the union of the $d$-dimensional faces of cubes $Q \in \mathcal{Q}_{1}(\rho)$, plus the interior of some $n$-1-dimensional faces that are not contained in a cube of $\mathcal{Q}_{1}(\rho)$, plus $\mathbb{R}^{n} \backslash D_{2}$. Thus (3.17) holds (for $\psi=\pi_{k} \circ \cdots \circ \pi_{1}$ ), and the other properties (3.15) and (3.16) are true by construction.

Variants of Lemma 3.14 are often used, where we also require that for $Q \in \mathcal{Q}(\rho), H^{d}(\psi(E \cap Q)) \leq C H^{d}(E \cap Q)$. Such a control is obtained by selecting more carefully the points $c_{F}$ in the various faces, with a nice Fubini argument. But for the moment we do not need such refinements.

Now we use Lemma 3.14 to construct a deformation $\left\{\psi_{t}\right\}$ of $E$ onto $E_{\rho}$. Set $\psi_{t}(y)=t y+(1-t) \psi(y)$ for $0 \leq t \leq 1$; this gives a one-parameter family of Lipschitz functions $\psi_{t}$, which all satisfy (3.15) and (3.16). In particular,

$$
\left|\psi_{t}(y)-y\right| \leq \sqrt{n} \rho \text { for } y \in \mathbb{R}^{n} \text { and } 0 \leq t \leq 1 .
$$

We may now return to our be a one-parameter family of deformations $\left\{\varphi_{t}\right\}_{0 \leq t \leq 1}$, as in the definition of $T N D$. Define new Lipschitz mappings $f_{t}, 0 \leq t \leq 1$, on $\mathbb{R}^{n}$ by

$$
\begin{cases}f_{t}(y)=\psi_{2 t}(y) & \text { for } 0 \leq t \leq 1 / 2 \\ f_{t}(y)=\varphi_{2 t-1}(\psi(y)) & \text { for } 1 / 2 \leq t \leq 1 .\end{cases}
$$

We want to check that $\left\{f_{t}\right\}$ satisfies the conditions (1.3)-(1.6), relative to the slightly larger ball $B=B\left(x,\left(1+\eta_{0}\right) r\right)$. First, each $f_{t}$ is clearly 
Lipschitz. Next, let us check that $f_{t}(\bar{B}) \subset \bar{B}$, as in (1.3). Observe that

$$
B(x, r) \subset D_{1} \subset D_{2} \subset B(x, r+2 \sqrt{n} \rho) \subset B\left(x, r+\alpha r_{1}\right) \subset B
$$

by definition of $D_{1}$ and $D_{2},(3.3),(3.11)$, and because $\alpha \leq \frac{\eta_{0}}{2}$ by (3.10).

If $y \in D_{2}$, then $\psi_{t}(y) \in D_{2}$ for all $t$, by (3.16), and so (3.20) says that $f_{s}(y) \in B$ for $s \leq 1 / 2$. If in addition $\psi(y) \in \bar{B}(x, r)$, then $\varphi_{t}(\psi(y)) \in$ $\bar{B}(x, r)$, by (1.3) for $\varphi_{t}$, and $f_{s}(y)=\varphi_{2 s-1}(\psi(y)) \in \bar{B}(x, r) \subset B$ for $s \geq 1 / 2$, by (3.20). If instead $\psi(y) \notin \bar{B}(x, r)$, then (1.5) says that $\varphi_{t}(\psi(y))=\psi(y)$, so $f_{s}(y)=\psi(y)$ for $s \geq 1 / 2$. Thus $f_{s}(y) \in D_{2} \subset B$, as for $s=1 / 2$ above. So $f_{t}\left(D_{2}\right) \subset B$, and we are left with $\bar{B} \backslash D_{2}$. But

$$
\psi_{t}(y)=y \text { and } f_{t}(y)=y \text { for all } t \text { when } y \in \mathbb{R}^{n} \backslash D_{2},
$$

by (3.15) (for $\psi_{t}$ ), and then (1.5) (because $\psi_{t}(y)=y$ lies out of $B(x, r)$, by (3.20)). Thus $f_{t}\left(\bar{B} \backslash D_{2}\right) \subset \bar{B}$ too, and $f_{t}$ satisfies (1.3).

Note that (1.4) (the continuity of $f_{t}(y)$ in $t$ ) holds because $\psi_{1}=\psi$ and $\varphi_{0}(y)=y$. Also, $f_{0}(y)=y$ because $\psi_{0}(y)=y$, and $f_{t}(y)=y$ for $y \notin B$, by (3.20) and (3.21). Which takes care of (1.5).

We still need to check (1.6). So let $y \in E \cap B$ and $0 \leq s \leq 1$ be given; we want to show that

$$
\operatorname{dist}\left(f_{s}(y), E\right) \leq \alpha_{0}\left(1+\eta_{0}\right) r .
$$

First,

$$
\operatorname{dist}\left(\psi_{t}(y), E\right) \leq|\psi(y)-y| \leq \sqrt{n} \rho \leq \frac{1}{2} \alpha r_{1} \leq \alpha r \leq \frac{1}{2} \alpha_{0} r
$$

by (3.18), (3.3), (3.11), and (3.10). So (3.22) already holds for $0 \leq s \leq$ $1 / 2$.

For $s>1 / 2$ we shall distinguish between cases again. Observe that

$$
\psi(y) \notin B(x, r) \text { when } y \in \mathbb{R}^{n} \backslash D_{1},
$$

because $y$ lies in a cube $Q \in \mathcal{Q}(\rho) \backslash \mathcal{Q}_{1}(\rho)$, and then (3.16) says that $\psi(y)$ lies in $Q$, which does not meet $B(x, r)$ because $B(x, r) \subset \operatorname{int}\left(D_{1}\right)$ (by (3.20) and because $B(x, r)$ is open).

If $y \in E \backslash D_{1}$ and $s \geq 1 / 2,(3.24)$ says that $\psi(y) \notin B(x, r)$, then $f_{s}(y)=\varphi_{2 s-1}(\psi(y))=\psi(y)$, by (1.5) for $\left\{\varphi_{t}\right\}$. Then (3.22) follows from (3.23).

We are left with the case when $y \in D_{1}$ (and $s \geq 1 / 2$ ). Let us first check that

$$
\psi(y) \in E_{\rho} \text { when } y \in E \cap D_{1} .
$$

Let $Q$ be a cube of $\mathcal{Q}_{1}(\rho)$ that contains $x$. Then $\psi(y) \in S_{d}(Q)$ by (3.17). Since $Q$ touches $E$ (because $x \in E), S_{d}(Q) \subset E_{\rho}$ and (3.25) follows. 
If in addition $\psi(y) \in B(x, r)$, we can apply (3.5) for $\left\{\varphi_{t}\right\}$ and get that $f_{s}(y)=\varphi_{2 s-1}(\psi(y))$ lies within $\alpha r_{1}$ of $E$. So (3.22) holds in that case, again because $\alpha r_{1} \leq \alpha_{0} r$. If $\psi(y) \notin B(x, r)$, then $f_{s}(y)=\psi(y)$ and (3.22) holds because of (3.23), just like when $s \leq 1 / 2$.

So $\left\{f_{t}\right\}$ satisfies (1.3)-(1.6). Since $E$ satisfies the hypotheses of Theorem 1.8, the analogue of $(1.7)$ for $B\left(x,\left(1+\eta_{0}\right) r\right)$ holds (because $(1+$ $\left.\eta_{0}\right) r \leq r_{0}$ by $(3.3)$ and $\left.(3.11)\right)$. That is,

$$
\begin{aligned}
H^{d}\left[B(x, r) \cap f_{1}(E)\right] \geq H^{d}\left[B \left(x,\left(1-\eta_{0}\right)(1+\right.\right. & \left.\left.\left.\eta_{0}\right) r\right) \cap f_{1}(E)\right] \\
& \geq \delta_{0}\left(\left(1+\eta_{0}\right) r\right)^{d} .
\end{aligned}
$$

On the other hand, we have already seen that $f_{1}(y)$ lies out of $B(x, r)$ when $y \in \mathbb{R}^{n} \backslash D_{1}$ (by (3.24) and (1.5) for $\left\{\varphi_{t}\right\}$ ). So

$$
B(x, r) \cap f_{1}(E) \subset f_{1}\left(E \cap D_{1}\right)=\left(\varphi_{1} \circ \psi\right)\left(E \cap D_{1}\right) \subset \varphi_{1}\left(E_{\rho}\right),
$$

where the last inclusion comes from (3.25). Hence

$$
H^{d}\left(B(x, r) \cap \varphi_{1}\left(E_{\rho}\right)\right) \geq \delta_{0} r^{d} \geq 2^{-d} r_{1}^{d} \delta_{0} \geq 2 \delta_{1} r_{1}^{d},
$$

by $(3.26),(3.11)$, and (3.10). And $H^{d}\left(\varphi_{1}\left(B(x, r) \cap E_{\rho}\right)\right)$ is even larger, since $\varphi_{1}(y)$ lies out of $B(x, r)$ when $y \notin B(x, r)$.

This completes our proof of (3.13); as was said before (3.13), we get that $E$ satisfies TND (as soon as it satisfies (3.9) and the topological condition in Theorem 1.8). Thus Theorem 1.8 follows from Theorem 3.8.

\section{A functional on unions of faces}

Let $E$ be a closed set that satisfies the hypotheses of Theorem 3.8. We want to prove that its Hausdorff dimension is at least $d^{*}>d$, and the main point will be to establish the following lemma.

Lemma 4.1. For every large $C_{1}>0$, we can find $\alpha_{2}>0$ and $r_{0}>0$ such that

$$
H^{d}\left(E_{\rho} \cap B\left(x_{1}, r_{1}\right)\right)>C_{1} r_{1}^{d}
$$

for all choices of $x_{1} \in E, r_{1} \in\left(0, r_{0}\right)$, and $\rho>0$ such that $\rho \leq \alpha_{2} r_{1}$.

We shall see in Section 7 that Theorem 3.8 is relatively easy and straightforward once we prove the lemma. And in fact it would be enough to prove the lemma for some (large) $C_{1}$ that can be computed from the dimension.

Our strategy for proving the lemma (which will keep us busy in Sections 4-6) will be to assume that (4.2) fails (so that (3.4) holds) and produce a contradiction. For this we shall use (3.4) and TND to construct an Ahlfors-regular, uniformly rectifiable set $F^{*}$ with a big intersection 
with $E_{\rho}$. The contradiction will then come from the fact that $F^{*}$ is fairly flat at most scales and locations (by uniform rectifiability), while our assumption (1.2) says that $E$ (and hence $E_{\rho}$, at least at scales larger than $\rho$ ) is never flat.

To find $F^{*}$, we shall minimize a functional like $J(F)=H^{d}\left(F \cap E_{\rho}\right)+$ $M H^{d}\left(F \backslash E_{\rho}\right)$, where $M$ is a large constant, and among sets $F$ that are a finite union $F^{*}$ of $d$-dimensional faces of cubes, plus a set of lower dimension $F \backslash F^{*}$, and that can be obtained from $E_{\rho}$ by deformations like the $\left\{\varphi_{t}\right\}$ in the definition of TND. The point of taking finite unions of faces is that the existence of a minimizer $F$ will be essentially trivial.

Our hypothesis $T N D$ will be used to find a ball $B(x, r) \subset B\left(x_{1}, r_{1}\right)$ where to define the functional, and then prove that $H^{d}(F)$ is never too small (by (3.6)). Then, if $M$ is large enough (compared to $\delta_{1}^{-1}$ ), we will get that $H^{d}\left(F^{*} \cap E_{\rho}\right)=H^{d}\left(F \cap E_{\rho}\right)$ is not too small either (because $H^{d}\left(F \backslash E_{\rho}\right)$ is very small $)$.

To show that $F^{*}$ is Ahlfors-regular and uniformly rectifiable, we shall prove that $F$ is a quasiminimal set (with the terminology of Almgren, a restricted set) in some open set, and then use the main result in [DS3]. The quasiminimality constant, and then the Ahlfors-regularity and uniform rectifiability constants will depend on $M$, hence essentially all the constants at hand (except $\alpha_{2}$ ), but we shall still be able to get a contradiction at some scale larger than $\rho$, if $\rho / r_{1}$ (or equivalently $\alpha_{2}$ ) is small enough.

A good part of the argument below is quite similar to parts of [DS3, Section 12], but with small differences that seem to make it hard to import the results of [DS3] directly. So we shall repeat some of the arguments here, mainly for the convenience of the reader.

We start with the preparation of an appropriate domain and the definition of a functional. Since we are given $C_{1}>0$, we get other constants $r_{0}, \alpha, \eta$ and $\delta_{1}$ from $T N D$. Let

$$
x_{1} \in E, 0<r_{1}<r_{0} \text {, and } 0<\rho \leq \alpha_{2} r_{1}
$$

be given, as in the statement of Lemma 4.1, and where $\alpha_{2}$ will be chosen near the end of the argument. We suppose that (4.2) fails (or equivalently that (3.4) holds) and we want a contradiction.

By $T N D$, we can find $x \in E$ and $r>0$ such that $B(x, r) \subset B\left(x_{1}, r_{1}\right)$ and (3.6) holds for all the appropriate deformations $\left\{\varphi_{t}\right\}$.

We want to define a domain $V \subset B(x, r)$ where things will really happen. Since we shall work with lots of slightly different security balls 
slightly smaller than $B(x, r)$, it will be convenient to set

$$
B_{j}=B\left(x, r-\eta r_{1}+\frac{j \eta r_{1}}{10}\right) \text { for } 0 \leq j \leq 10 .
$$

Recall that $\eta r_{1} \leq r$ (because (3.6) holds for the identity mapping, see the comment a little below (3.7)). So there is no difficulty with the definitions in (4.4). Also observe that $B_{0} \subset \cdots \subset B_{10}$, and that the annulus from (3.7) is

$$
A_{\eta r_{1}}(x, r)=B(x, r) \backslash B_{0}=B_{10} \backslash B_{0} .
$$

Things will be easier if the domain $V$ is composed of (multiples) of dyadic cubes. First choose a number $L$ such that

$$
\frac{1}{200} \operatorname{Min}(\eta, \alpha) r_{1} \leq \sqrt{n} L \leq \frac{1}{100} \operatorname{Min}(\eta, \alpha) r_{1},
$$

and such that $\rho^{-1} L$ is a power of 2 . Call $\mathcal{Q}(L)$ the set of "dyadic cubes" of sidelength $L$, as in (3.1). Set

$$
\begin{aligned}
& \mathcal{Q}_{D}=\left\{Q \in \mathcal{Q}(L) ; Q \cap B_{6} \neq \emptyset\right\}, \\
& \mathcal{Q}_{V}=\left\{Q \in \mathcal{Q}_{D} ; \operatorname{dist}(Q, E) \leq \frac{\alpha r_{1}}{2}\right\}, \\
& D=\bigcup_{Q \in \mathcal{Q}_{D}} Q, \text { and } V=\bigcup_{Q \in \mathcal{Q}_{V}} Q .
\end{aligned}
$$

Notice that $\operatorname{diam}(Q)=\sqrt{n} L \leq 100^{-1} \operatorname{Min}(\eta, \alpha) r_{1}$ for $Q \in \mathcal{Q}(L)$, by $(4.6)$, so

$$
B_{6} \subset D \subset B_{7} \subset B(x, r)
$$

and also

$$
\operatorname{dist}(y, E) \leq \frac{\alpha r_{1}}{2}+\sqrt{n} L<\alpha r_{1} \text { for } y \in V .
$$

Let us already decide that we shall take $\alpha_{2}<(200 \sqrt{n})^{-1} \operatorname{Min}(\eta, \alpha)$, to make sure that in the following construction,

$$
\rho \leq L
$$

by (4.6). Note that each cube of $\mathcal{Q}(\rho)$ is neatly contained in a cube of $\mathcal{Q}(L)$, because $\rho^{-1} L$ is a power of 2 . We shall work for a long time with a given $\rho$, but it will be important to make sure that our various constants do not depend on $\rho$ (or $\alpha_{2}$ ), because our final contradiction will require $\rho$ to be small enough compared to $r_{1}$.

Consider the class $\mathcal{F}_{0}$ of closed subsets of $D$ that are a (finite) union of $d$-dimensional faces of cubes $Q \in \mathcal{Q}(\rho)$, plus perhaps a set of Hausdorff 
dimension $\leq d-1$. For $F \in \mathcal{F}_{0}$, denote by $F^{*}$ the union of the faces of dimension $d$ (of cubes in $\mathcal{Q}(\rho)$ ) that are contained in $F$. Thus

$$
F^{*} \subset F \text { and } \mathcal{H} \operatorname{dim}\left(F \backslash F^{*}\right) \leq d-1 .
$$

We are mostly interested in the subclass $\mathcal{F}$ of the sets $F \in \mathcal{F}_{0}$ that can be written

$$
F=\varphi_{1}\left(E_{\rho} \cap D\right),
$$

where $\left\{\varphi_{t}\right\}, 0 \leq t \leq 1$, is a family of Lipschitz mapping on $\mathbb{R}^{n}$ such that

$$
\varphi_{t}(D) \subset D \text { for all } t
$$

$$
\begin{aligned}
& \varphi_{t}(y) \text { is a continuous function of } t \text { for every } y \in \mathbb{R}^{n}, \\
& \varphi_{t}(y)=y \text { for } t=0 \text { and for } y \in \mathbb{R}^{n} \backslash D, \\
& \operatorname{dist}\left(\varphi_{t}(y), E\right)<\alpha r_{1} \text { for } y \in E_{\rho} \cap D \text { and } 0 \leq t \leq 1,
\end{aligned}
$$

and even

$$
\varphi_{1}(y) \in V \text { for } y \in E_{\rho} \cap D .
$$

Note that even though the set $F^{*} \backslash F$ has a lower Hausdorff dimension, it may play an important role in the description above. That is, (4.14) gives a representation of $F$ as a deformation of $E_{\rho} \cap D$, but we do not necessarily have a similar representation of $F^{*}$, because for instance we cannot always deform $F$ onto $F^{*}$.

Let us check that $\mathcal{F}$ is not empty, and that

$$
E_{\rho} \cap D \in \mathcal{F} .
$$

The point is that we can take the trivial deformation $\varphi_{t}(y)=y$ in (4.15)(4.19). Of course only (4.18) and (4.19) need to be checked. But

$$
\operatorname{dist}(y, E) \leq \sqrt{n} \rho \leq \sqrt{n} L \leq 100^{-1} \alpha r_{1} \text { for } y \in E_{\rho},
$$

by definition of $E_{\rho},(4.12)$ and (4.6). Hence

$$
E_{\rho} \cap D=E_{\rho} \cap V,
$$

by (4.8). So(4.18) and (4.19) hold, $\varphi_{t}(y)=y$ satisfies the required conditions, and $E_{\rho} \cap D \in \mathcal{F}$ (because it is a union of $d$-dimensional faces of cubes from $\mathcal{Q}(\rho))$.

Let us use TND. Each family $\left\{\varphi_{t}\right\}$ as in (4.15)-(4.19) satisfies (1.3), (1.4), and (1.5), because $D \subset B(x, r)$ (by (4.10)). Let us check that it also satisfies (3.5), i.e., that $\operatorname{dist}\left(\varphi_{t}(y), E\right) \leq \alpha r_{1}$ for $y \in E_{\rho} \cap B(x, r)$ and $t \in[0,1]$. If $y$ lies out of $D$, then $\varphi_{t}(y)=y$, hence $\varphi_{t}(y) \in E_{\rho}$ and the result follows from (4.21). Otherwise, we can simply apply (4.18). 
So (3.5) holds, we can apply TND, and (3.6) says that

$$
H^{d}\left(\varphi_{1}\left(E_{\rho} \cap B(x, r)\right)\right) \geq \delta_{1} r_{1}^{d}+H^{d}\left(E_{\rho} \cap A_{\eta r_{1}}(x, r)\right) .
$$

Set $H=E_{\rho} \cap[B(x, r) \backslash D]$ for a few lines. Then $\varphi_{1}\left(E_{\rho} \cap B(x, r)\right)=$ $\varphi_{1}\left(E_{\rho} \cap D\right) \cup \varphi_{1}(H)$ (because $D \subset B(x, r)$, see (4.10)), and this union is disjoint because $\varphi_{1}(D) \subset D$ and $\varphi_{1}(y)=y \notin D$ when $y$ lies out of $D$. Moreover, $\varphi_{1}(H)=H$ (because $\varphi_{1}(y)=y$ on $H$ ), so the left-hand side of $(4.23)$ is $H^{d}\left(\varphi_{1}\left(E_{\rho} \cap D\right)\right)+H^{d}(H)$. Also,

(4.24) $H=E_{\rho} \cap[B(x, r) \backslash D] \subset E_{\rho} \cap\left[B(x, r) \backslash B_{6}\right] \subset E_{\rho} \cap A_{\eta r_{1}}(x, r)$ by (4.10) and (4.5) (or the definition (3.7)). So we can subtract $H^{d}(H)$ from both sides of (4.23), and we get that

$$
H^{d}\left(\varphi_{1}\left(E_{\rho} \cap D\right)\right) \geq \delta_{1} r_{1}^{d}+H^{d}\left(E_{\rho} \cap A_{\eta r_{1}}(x, r) \cap D\right) .
$$

This holds for every family $\left\{\varphi_{t}\right\}$ such that (4.15)-(4.19) hold. When we restrict to families where $F=\varphi_{1}\left(E_{\rho} \cap D\right) \in \mathcal{F}_{0}$ as in (4.14), we get that

$$
\begin{aligned}
H^{d}(F)=H^{d}\left(\varphi_{1}\left(E_{\rho} \cap D\right)\right) & \\
& \geq \delta_{1} r_{1}^{d}+H^{d}\left(E_{\rho} \cap A_{\eta r_{1}}(x, r) \cap D\right) \text { for } F \in \mathcal{F} .
\end{aligned}
$$

Set $M=\frac{2 C_{1}}{\delta_{1}}$, with $C_{1}$ as in (4.2) and (3.4), and define a functional on $\mathcal{F}$ by

$$
J(F)=H^{d}\left(F \cap E_{\rho}\right)+M H^{d}\left(F \backslash E_{\rho}\right) \text { for } F \in \mathcal{F} .
$$

Note that $J(F)=H^{d}\left(F^{*} \cap E_{\rho}\right)+M H^{d}\left(F^{*} \backslash E_{\rho}\right)$, where $F^{*}$ still denotes the finite union of $d$-faces that satisfies (4.13). Since $\mathcal{F} \neq \emptyset$ (by $(4.20)$ ) and there is only a finite number of possible sets $F^{*}$, we can find a minimizer $F$, i.e., a set $F \in \mathcal{F}$ such that

$$
J(F)=\inf \left\{J\left(F^{\prime}\right) ; F^{\prime} \in \mathcal{F}\right\} .
$$

Recall from (4.20) that $F^{\prime}=E_{\rho} \cap D \in \mathcal{F}$. Hence

(4.29) $J(F) \leq J\left(E_{\rho} \cap D\right)=H^{d}\left(E_{\rho} \cap D\right) \leq H^{d}\left(E_{\rho} \cap B(x, r)\right) \leq C_{1} r_{1}^{d}$,

by (4.10) and because we assumed that (4.2) does not hold (to get a contradiction). Then

$$
H^{d}\left(F \backslash E_{\rho}\right) \leq M^{-1} J(F) \leq \frac{\delta_{1}}{2 C_{1}} C_{1} r_{1}^{d}=\frac{\delta_{1} r_{1}^{d}}{2}
$$

by (4.27), (4.29), and our definition of $M$. Now (4.26) yields

$$
\begin{aligned}
H^{d}\left(F \cap E_{\rho}\right) \geq H^{d}(F)- & H^{d}\left(F \backslash E_{\rho}\right) \\
& \geq \delta_{1} r_{1}^{d} / 2+H^{d}\left(E_{\rho} \cap A_{\eta r_{1}}(x, r) \cap D\right) .
\end{aligned}
$$


Thus $F$ has a fairly big intersection with $E_{\rho}$. It will be useful to have a lower bound on the size of the part of $F \cap E_{\rho}$ that lies in $B_{0}=$ $B\left(x, r-\eta r_{1}\right)$ (recall the notation (4.4)), because it will be easier to show that $F^{*}$ is regular there. Note that $F \cap E_{\rho}$ is the disjoint union of $F \cap E_{\rho} \cap B_{0}$ and $F \cap E_{\rho} \backslash B_{0}$. Recall that $F \subset D \subset B(x, r)$ by definition of $\mathcal{F}_{0}$ and (4.10), so $F \cap E_{\rho} \backslash B_{0} \subset E_{\rho} \cap A_{\eta r_{1}}(x, r) \cap D$, by (4.5). So we may subtract $H^{d}\left(F \cap E_{\rho} \backslash B_{0}\right)$ from both sides of (4.31) and we get that

$$
H^{d}\left(F \cap E_{\rho} \cap B_{0}\right) \geq \delta_{1} r_{1}^{d} / 2 .
$$

Our next goal is to show that $F^{*}$ is locally uniformly rectifiable in $B_{0}$ because $F$ is a minimizer for some nice functional. This will be used to produce lots of balls where $F$ (and hence $E_{\rho}$ ) are very flat, and a contradiction with (1.2) will ensue.

\section{Almgren quasiminimality of $F$}

Let $F$ be the minimizer for $J$ introduced in the last section, and set

$$
B_{2}=B\left(x, r-\frac{8 \eta r_{1}}{10}\right)
$$

(as in (4.4)). The goal of this section is to show that

(5.2) $S=F \cap B_{2}$ is a $\left(B_{2}, k, \delta\right)$-quasiminimizer for $H^{d}$,

where $k$ is a constant that depends on $C_{1}$ and $\delta_{1}$ (in fact, $k=C M=$ $2 C C_{1} \delta_{1}^{-1}$ for some geometric constant $C$ ),

$$
\delta=4^{-n-1} L,
$$

and the notion of quasiminimizer for $H^{d}$ is the same as in [DS3] and $[\mathbf{A l}]$, and will be explained soon. See [DS3, pp. 7-8], for the official definition that we shall use.

What does (5.2) mean? First, $S$ should be closed in $B_{2}$ and non empty (Condition (1.1) in [DS3]). This comes from (4.14) and (4.32) respectively. Next, $H^{d}(S)$ should be locally finite (Condition (1.2) in [DS3]), which is obvious because $H^{d}(F) \leq J(F)<+\infty$. And then $S$ should satisfy (1.8) in [DS3].

For this, we give ourselves a Lipschitz function $\psi: \mathbb{R}^{n} \rightarrow \mathbb{R}^{n}$ and that satisfies the conditions (1.5)-(1.7) in [DS3]. In particular, (1.5) and (1.6) says that if we set

$$
W=\left\{z \in \mathbb{R}^{n} ; \psi(z) \neq z\right\},
$$

then

$$
\operatorname{diam}(W \cup \psi(W))<\delta
$$


and

$$
W \cup \psi(W) \Subset B_{2},
$$

i.e., $\operatorname{dist}\left(W \cup \psi(W), \mathbb{R}^{n} \backslash B_{2}\right)>0$. The condition (1.7) in [DS3] says that there is a homotopy from the identity to $f$, with conditions like (5.5) and (5.6), but we shall not need it. We need to show that under these conditions,

$$
H^{d}(S \cap W) \leq k H^{d}(\psi(S \cap W)) .
$$

Our strategy for proving this is to use $\psi$ to construct a competitor $F_{1} \in \mathcal{F}$, and then deduce (5.7) from the minimality of $J(F)$. Of course we cannot take $F_{1}=\psi(F)$ directly, because it probably does not lie in $\mathcal{F}$, so we shall have to modify it in two ways.

First, it could be that $\psi(F)$ gets out of $V$, which would be bad because of our constraint (4.19) on deformations. To fix this problem, we shall construct a Lipschitz retraction onto $V$, and use it to push back $\psi(F)$ inside $V$.

But also, $\psi(F)$ is not necessarily a finite union of $d$-faces, plus a set of lower dimension, so we shall need to project it onto $d$-dimensional skeletons (a little like in Lemma 3.14).

We start with the Lipschitz retraction.

Lemma 5.8. If $V$ is a finite union of (closed) dyadic cubes of sidelength $L$, there is $4^{n}$-Lipschitz function $h$ defined on

$$
V_{+}=\left\{y \in \mathbb{R}^{n} ; \operatorname{dist}(y, V) \leq \frac{L}{4}\right\},
$$

and such that

$$
\begin{aligned}
& h\left(V_{+}\right) \subset V, \\
& h(y)=y \quad \text { for } y \in V,
\end{aligned}
$$

and

$$
|h(y)-y| \leq \sqrt{n} L \quad \text { for } y \in V_{+} .
$$

The statement is not exactly the same as in Lemma 12.2 in [DS3], but the proof will be the same. We shall get $h$ as a composition of a few mappings $g_{j}$

Set $g_{1}(y)=y$ on $V$. Call $\mathcal{Q}_{+}$the collection of cubes $Q \in \mathcal{Q}(L)$ that touch $V$ but are not contained in it. Thus

$$
V_{+} \backslash V \subset \bigcup_{Q \in \mathcal{Q}_{+}} Q \cap V_{+} .
$$


Call $c_{Q}$ the center of $Q \in \mathcal{Q}_{+}$. Then $\operatorname{dist}\left(c_{Q}, V_{+}\right) \geq \operatorname{dist}\left(c_{Q}, V\right)-L / 4 \geq$ $L / 4$. We define $g_{1}$ on $Q \cap V_{+}$to be the radial projection on $\partial Q$ with center $c_{Q}$ (as defined in the proof of Lemma 3.14). Note that $g_{1}(y)=y$ on $\partial Q$, so our different definitions coincide (on intersections of cubes). Observe also that $g_{1}$ is 4 -Lipschitz on $V_{+}$, because each $c_{Q}$ if far from $V_{+}$. Finally, we claim that

$$
\operatorname{dist}\left(g_{1}(y), V\right) \leq \operatorname{dist}(y, V) \leq 4^{-1} L \text { for } y \in V_{+} .
$$

This is trivial when $y \in V$. Then let $y \in V_{+} \backslash V$ be given, let $Q \in \mathcal{Q}_{+}$ contain $y$, and let $z$ be a point of $V$ such that $|z-y|=\operatorname{dist}(y, V)$. Also call $Q_{z}$ a cube of $V$ that contains $z$. Then $Q_{z}$ touches $Q$ (because otherwise it would be too far), and even $z \in Q \cap Q_{z}$ (by inspection). Note that $c_{Q}$ lies further from $T=Q \cap Q_{z}$ than $y$. Then $\operatorname{dist}\left(g_{1}(y), Q\right) \leq$ $\operatorname{dist}\left(g_{1}(y), T\right) \leq \operatorname{dist}(y, T) \leq|z-y|=\operatorname{dist}(y, V)$, as needed for (5.14). Now (5.14) yields

$$
g_{1}\left(V_{+}\right) \subset V \cup \bigcup_{Q \in \mathcal{Q}_{+}}\left[S_{n-1}(Q) \cap V_{+}\right] .
$$

We set $g_{2}(y)=y$ on $V$. For each $(d-1)$-face $T$ of a cube $Q \in \mathcal{Q}_{+}$ such that $T$ is not already contained in $V$, we decide that for $y \in T \cap V_{+}$, $g_{2}(y)$ is the radial projection of $y$ on $\partial T$ (centered at the center of $T$ ). This definition is coherent, because $T$ only meets different faces or $V$ along $\partial T$, where $g_{2}$ is the identity. Note that we do not need to extend $g_{2}$, because it is already defined on $g_{1}\left(V_{+}\right)$, by (5.15). Also, $g_{2}$ is 4-Lipschitz, again because $y \in V_{+}$is never close to the center of $T$.

As before, $\operatorname{dist}\left(g_{2}(y), V\right) \leq \operatorname{dist}(y, V) \leq 4^{-1} L$ for $y \in T \cap V_{+}$, and hence $g_{2}(y) \in S_{n-2}(T) \cap V_{+}$. Thus

$$
g_{2} \circ g_{1}\left(V_{+}\right) \subset V \cup \bigcup_{Q \in \mathcal{Q}_{+}}\left[S_{n-2}(Q) \cap V_{+}\right] .
$$

We can define $g_{3}$ on $V$ and on the sets $T \cap V_{+}$, where $T$ is a $(n-2)$ dimensional face of some cube $Q \in \mathcal{Q}_{+}$and is not contained in $V$, as we did before.

After a few iterations, we get a $4^{n}$-Lipschitz mapping $h=g_{1} \circ \cdots \circ g_{n}$ that sends $V_{+}$to $V$, plus the intersection of $V_{+}$with a set of vertices. But only the vertices of $V$ can lie in $V^{+}$, so $h\left(V_{+}\right) \subset V$, as in (5.10). We have that $h(y)=y$ for $y \in V$ because all the $g_{j}$ fix points of $V$, and our last condition (5.12) holds because $g_{j}(Q) \subset Q$ for each $Q \in \mathcal{Q}(L)$ and each $j$, so that if we start from $y$ in some cube $Q \in \mathcal{Q}(L), h(y)$ lies in the same $Q$. 
We may now return to the Lipschitz mapping $\psi$ of the beginning of this section. Recall from (4.14) and (4.19) that

$$
F=\varphi_{1}\left(E_{\rho} \cap D\right) \subset V
$$

for some family $\left\{\varphi_{t}\right\}$ that satisfies (4.15)-(4.19). Notice that if $\psi(y) \neq y$, then $y \in W$ (by (5.4)), and then $|\psi(y)-y| \leq \operatorname{diam}(W \cup \psi(W))<\delta$ by (5.5). Hence

$$
|\psi(y)-y| \leq \delta=4^{-n-1} L \text { for } y \in \mathbb{R}^{n},
$$

by (5.3). By (5.17) and (5.18), $\psi(y) \in V_{+}$(and hence $h(\psi(y))$ is defined) for $y \in F$. Set

$$
F_{1}=\psi(F) \text { and } F_{2}=h\left(F_{1}\right) .
$$

Thus $F_{2} \subset V$. We still need to project it back on $d$-dimensional skeletons. For this part of the construction, we shall work on cubes of sidelength $\rho$. Some additional notation will be useful. Call $\mathcal{S}_{d} \subset \mathbb{R}^{n}$ the union of all the $d$-dimensional faces of cubes $Q \in \mathcal{Q}(\rho)$. With the notations of the beginning of Section 3,

$$
\mathcal{S}_{d}=\bigcup_{Q \in \mathcal{Q}(\rho)} S_{d}(Q) .
$$

For $A \subset \mathbb{R}^{n}$, call $\mathcal{N}(A)$ the union of all the cubes $Q \in \mathcal{Q}(\rho)$ which meet a cube of $\mathcal{Q}(\rho)$ which meets $A$.

Let $W$ be as in (5.4), and set

$$
X=h(\psi(F \cap W)) .
$$

This is the part that we may need to project back onto $\mathcal{S}_{d}$. Indeed

$$
h(\psi(y))=\psi(y)=y \text { for } y \in F \backslash W,
$$

by (5.4), (5.17), and (5.11), so $h(\psi(F \backslash W))=F \backslash W$. Except for a set of lower dimension, this set is contained in $F^{*}$, which itself is contained in $\mathcal{S}_{d}$ by definition of $\mathcal{F}_{0}$.

We may now apply Lemma 11.14 on [DS3, p. 90] to $X$. The notations are almost the same, except that $\rho$ is called $2^{-j}$ there, and what we call $\mathcal{N}(A)$ here is denoted by $\mathcal{N}_{j}(A)$ there. We get the existence of a Lipschitz 
function $f$ on $\mathbb{R}^{n}$ such that

$$
\begin{gathered}
f(z)=z \text { out of } \mathcal{N}(\bar{X}), \\
f(z)=z \text { for } z \in \mathcal{S}_{d}, \\
f(\bar{X}) \subset \mathcal{S}_{d}, \\
f(Q) \subset Q \text { for } Q \in \mathcal{Q}(\rho),
\end{gathered}
$$

and

$$
H^{d}\left(f\left(\bar{X} \cap Q \backslash \mathcal{S}_{d}\right)\right) \leq C H^{d}\left(\bar{X} \cap Q \backslash \mathcal{S}_{d}\right) \text { for every } Q \in \mathcal{Q}(\rho) .
$$

The proof of Lemma 11.14 in [DS3] is similar to the proof of Lemma 3.14 above, except that we also need (5.27) now. As was alluded to above, this can be obtained by choosing the centers $c_{F}$ in the various faces more carefully, so that their average distance to the points of the current image of $\bar{X}$ is not too small.

Set $F_{3}=f\left(F_{2}\right)=f(h(\psi(F)))$, and let us already check that

$$
\mathcal{H} \operatorname{dim}\left(F_{3} \backslash \mathcal{S}_{d}\right) \leq d-1 .
$$

We already know from (5.22) that $h\left(\psi\left(F^{*} \backslash W\right)\right)=F^{*} \backslash W \subset \mathcal{S}_{d}$, because $F^{*} \subset \mathcal{S}_{d}$ by definition of $\mathcal{F}_{0}$. Then $f\left(h\left(\psi\left(F^{*} \backslash W\right)\right)\right) \subset \mathcal{S}_{d}$ too, by (5.24). Since $f\left(h\left(\psi\left(F^{*} \cap W\right)\right)\right) \subset f(X) \subset \mathcal{S}_{d}$ by definition (5.21) of $X$ and by (5.25), we are left with $f\left(h\left(\psi\left(F \backslash F^{*}\right)\right)\right)$, which has Hausdorff dimension at most $d-1$ by (4.13) and because $f \circ h \circ \psi$ is Lipschitz.

Now (5.28) does not exactly say that $F_{3} \in \mathcal{F}_{0}$, because $F_{3} \cap \mathcal{S}_{d}$ is not necessarily an exact union of full $d$-faces. To fix this, we need to compose with yet another function $\pi$.

Call $\mathcal{A}$ the set of $d$-dimensional faces of cubes in $\mathcal{Q}(\rho)$ that meet $F_{3}$ on a set of dimension $>d-1$ but are not contained in $F_{3}$. For $T \in \mathcal{A}$, choose an origin $c_{T} \in \operatorname{int}(T) \backslash F_{3}$. Such a point exists by definition of $\mathcal{A}$, and $\operatorname{dist}\left(c_{T}, F_{3}\right)>0$ because $F_{3}$ is closed. For $z \in F_{3} \cap T$, we let $\pi(z)$ be the radial projection on $\partial T$ with center $c_{T}$. Then we extend this definition to $T$, so that $\pi$ is Lipschitz on $T, \pi(T) \subset T$, and $\pi(z)=z$ on $\partial T$. We do this for each $T \in \mathcal{A}$, then set $\pi(z)=z$ on the rest of $\mathcal{S}_{d}$, and then extend $\pi$ to $\mathbb{R}^{n}$ so that it stays Lipschitz,

$$
\pi(Q) \subset Q \text { for every } Q \in \mathcal{Q}(\rho)
$$

and that

(5.30) $\pi(z)=z$ on the cubes that do not touch a face $T \in \mathcal{A}$.

The details are the same as in the previous similar constructions. 
Our next middle-term goal is to show that

$$
F_{4}=\pi\left(F_{3}\right)=\pi \circ f \circ h \circ \psi(F)
$$

lies in the set $\mathcal{F}$ of competitors for $J$.

By definition of $\pi, \pi\left(F_{3}\right)$ is now an exact union of $d$-faces (precisely, the faces of $\mathcal{S}_{d}$ that were contained in $F_{3}$ ), plus perhaps a set of dimension $\leq d-1$ coming from the almost-empty faces, and the boundaries of faces $T \in \mathcal{A}$ where we used $\pi$ to project. And the remaining piece $\pi\left(F_{3} \backslash \mathcal{S}_{d}\right)$ is still at most $(d-1)$-dimensional, by (5.28). So we shall get that $F_{4} \in \mathcal{F}_{0}$ as soon as we check that $F_{4} \subset D$.

Recall that $F_{2}=h\left(F_{1}\right) \subset V$, by (5.19) and (5.10). Since the mappings $f$ and $\pi$ preserve cubes of $\mathcal{Q}(\rho)$ (by (5.26) and (5.29)), $F_{4}=$ $\pi\left(f\left(F_{2}\right)\right) \subset V \subset D$ (by (4.8) and (4.9)), and

$$
F_{4} \in \mathcal{F}_{0} .
$$

The verification that $F_{4} \in \mathcal{F}$ will take some time, because we need to construct a family of Lipschitz functions as in (4.14)-(4.19). The following function $g$, which we first define on $V$, will be useful. Set

$$
g(z)=\pi \circ f \circ h \circ \psi(z) \text { for } z \in V .
$$

The definition makes sense, because if $z \in V$, then $\operatorname{dist}(\psi(z), V)<$ $4^{-n} L$ by (5.18), and then $h(\psi(z))$ is defined and lies in $V$, by $(5.9)$ and (5.10). Then $g(z)$ is well defined, because $f$ and $\pi$ are defined everywhere. Note that in addition

$$
g(z) \in V \subset D \text { for } z \in V
$$

because $f$ and $\pi$ preserve cubes of $\mathcal{Q}(\rho)$, and $V \subset D$ by definition (see (4.8) and (4.9)).

We want to see how much $g$ moves points. First observe that

$$
h(\psi(z))=\psi(z)=z \text { for } z \in V \backslash B_{2},
$$

by (5.4), (5.6), and (5.11). Even when $z \in B_{2}$ we can still say something, since

$$
\begin{aligned}
& |h(\psi(z))-z| \leq|h(\psi(z))-\psi(z)|+|\psi(z)-z| \\
& \leq \sqrt{n} L+4^{-n-1} L \leq 2 \sqrt{n} L \text { for } z \in V,
\end{aligned}
$$

by (5.12) and (5.18). 
Next we look at $f$ and the set $\mathcal{N}(\bar{X})$ where it may move points. Recall from (5.21) that $X=h(\psi(F \cap W))$, which is defined because $F \subset V$ (by (5.17)). Also recall from (5.4) that $\psi(W) \subset B_{2}$, so

(5.37) $\operatorname{dist}\left(h(\psi(z)), B_{2}\right) \leq|h(\psi(z))-\psi(z)| \leq \sqrt{n} L$ for $z \in F \cap W$,

by (5.12) again. In other words, $\operatorname{dist}\left(y, B_{2}\right) \leq \sqrt{n} L$ for $y \in X$.

By definition, every point of $\mathcal{N}(\bar{X})$ lies within $2 \sqrt{n} \rho$ of $X$, hence within $3 \sqrt{n} L$ of $B_{2}$ (by (4.12)). Since $3 \sqrt{n} L<\eta r_{1} / 30$ by (4.6), we get that

$$
\mathcal{N}(\bar{X}) \subset B_{3} .
$$

Recall from (5.23) that $f(z)=z$ out of $\mathcal{N}(\bar{X})$. Then

$$
f(h(\psi(z)))=h(\psi(z))=\psi(z)=z \text { for } z \in V \backslash B_{3},
$$

by (5.35), and (5.38).

Now we consider $\pi$. Recall from (5.30) that $\pi(z)=z$ unless $z \in Q$ for a cube $Q \in \mathcal{Q}(\rho)$ that touches a face $T \in \mathcal{A}$. Let us check that

$$
\operatorname{dist}\left(T, B_{3}\right) \leq \frac{\eta r_{1}}{30} \text { for } T \in \mathcal{A} .
$$

Let $T \in \mathcal{A}$ be given, and first assume that $T \subset F$. Since $T$ is not contained in $F_{3}=f(h(\psi(F)))$, it must contain some point $z \in F$ such that $f(h(\psi(z))) \neq z$. Since $z \in F \subset V,(5.39)$ says that $z \in B_{3}$. In this case (5.40) holds trivially.

If $A$ is not contained in $F, \mathcal{H} \operatorname{dim}(A \cap F) \leq d-1$ (because $F \in \mathcal{F}_{0}$ ), hence there are some points of $F_{3} \cap T$ that do not lie in $F$. Such points are of the form $y=f(h(\psi(z)))$, with $z \in F$. Since $y \notin F, f(h(\psi(z))) \neq z$ and (5.39) says that $z \in B_{3}$. Also,

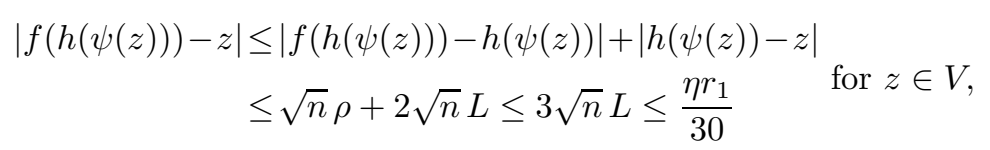

by (5.26), (5.36), (4.12), and (4.6). When we apply this with $z \in F$ and $y=f(h(\psi(z))) \in F_{3} \cap T$ as above, we get that $\operatorname{dist}\left(T, B_{3}\right) \leq|z-y|=$ $|z-f(h(\psi(z)))| \leq \eta r_{1} / 30$, and (5.40) holds.

If $z \in \mathbb{R}^{n}$ is such that $\pi(z) \neq z,(5.30)$ says that $z \in Q$ for a cube $Q \in$ $\mathcal{Q}(\rho)$ that touches a face $T \in \mathcal{A}$; then $\operatorname{dist}\left(z, B_{3}\right) \leq \eta r_{1} / 30+2 \sqrt{n} \rho<$ $\eta r_{1} / 10$ by (5.40), (4.12), and (4.6). Thus

$$
\pi(z)=z \text { for } z \in \mathbb{R}^{n} \backslash B_{4},
$$

and hence (5.39) and (5.33) yield

$$
g(z)=f(h(\psi(z)))=h(\psi(z))=\psi(z)=z \text { for } z \in V \backslash B_{4} .
$$


We are now ready to complete our definition of $g$.

Lemma 5.44. We can find a Lipschitz function $g: \mathbb{R}^{n} \rightarrow \mathbb{R}^{n}$ such that (5.33) holds,

$$
g(z)=z \quad \text { for } z \in \mathbb{R}^{n} \backslash B_{5}
$$

and

$$
g(z) \in \bar{B}_{5} \subset D \quad \text { for } z \in \bar{B}_{5} .
$$

Set $H=\left[V \cap B_{5}\right] \cup\left[\partial B_{5}\right]$. If we keep (5.33) on $V \cap B_{5}$ and set $g(z)=z$ on $\partial B_{5}$, we have a coherent definition of $g$ on $H$, by (5.43). Moreover, $g$ is Lipschitz on $H$. Indeed, since $g$ is clearly Lipschitz on $V$ (by (5.33)) and on $\bar{B}_{5} \backslash B_{4}$ (because it is the identity there), it is enough to estimate $g(y)-g(z)$ when $y \in V \cap B_{4}$ and $z \in \partial B_{5}$. But then $|g(y)-g(z)| \leq 2 r_{1}$ because $g(y) \in V \subset B(x, r)$ (by (5.34) and (4.4)), while $|y-z| \geq \eta r_{1} / 10$ (by (4.4) again). So $g$ is Lipschitz on $H$.

Let us also check that $g(H) \subset \bar{B}_{5}$. When $z \in\left[V \backslash B_{4}\right] \cup \partial B_{5}, g(z)=z$ (by (5.43)), and so $g(z) \in \bar{B}_{5}$. Otherwise, notice that for $z \in V$,

$$
\begin{aligned}
|g(z)-z| & \leq|g(z)-f(h(\psi(z)))|+|f(h(\psi(z)))-z| \\
& \leq|\pi(f(h(\psi(z))))-f(h(\psi(z)))|+3 \sqrt{n} L \\
& \leq \sqrt{n} \rho+3 \sqrt{n} L<4 \sqrt{n} L \leq \frac{\eta r_{1}}{20}
\end{aligned}
$$

by (5.33), (5.41), (5.29), (4.12), and (4.6). In the remaining case when $z \in V \cap B_{4}$, we still get that $g(z) \in \bar{B}_{5}$.

It is now easy to extend $g$ from $H$ to $\bar{B}_{5}$, so that it stays Lipschitz and with values in $\bar{B}_{5}$. [Otherwise, compose with a Lipschitz retraction on $\bar{B}_{5}$.] Finally extend $g$ to the rest of $\mathbb{R}^{n}$, by setting $g(z)=z$ out of $\bar{B}_{5}$. We still get a Lipschitz function, and the properties (5.33), (5.45), and (5.46) hold by construction. Lemma 5.44 follows.

Recall that we want to use $g$ to construct a family of Lipschitz functions as in (4.14)-(4.19) and show that $F_{4} \in \mathcal{F}$. Denote by $\left\{\varphi_{t}\right\}$ the family associated to $F \in \mathcal{F}$ and set

$$
\widetilde{\varphi}_{t}(y)=\varphi_{2 t}(y) \text { for } 0 \leq t \leq 1 / 2
$$

(and all $y \in \mathbb{R}^{n}$ ) and

$$
\widetilde{\varphi}_{t}(y)=(2-2 t) \varphi_{1}(y)+(2 t-1) g\left(\varphi_{1}(y)\right) \text { for } 1 / 2<t \leq 1 .
$$

Each $\widetilde{\varphi}_{t}$ is Lipschitz, because $g$ and the $\varphi_{t}$ are. Next we need to check that $\widetilde{\varphi}_{t}(D) \subset D$, as in (4.15). When $t \leq 1 / 2$, this comes directly from (4.15) (for the $\varphi_{t}$ ). So we may restrict to $t>1 / 2$. 
Let $y \in D$ be given. If $\varphi_{1}(y) \in \mathbb{R}^{n} \backslash B_{5}$, (5.45) says that $g\left(\varphi_{1}(y)\right)=$ $\varphi_{1}(y)$ and hence $\widetilde{\varphi}_{t}(y)=\varphi_{1}(y) \in D$ (by (5.49) and (4.15) for $\left.\varphi_{1}\right)$. Otherwise $\varphi_{1}(y) \in B_{5},(5.46)$ says that $g\left(\varphi_{1}(y)\right) \in \bar{B}_{5}$, and $\widetilde{\varphi}_{t}(y) \in$ $\bar{B}_{5} \subset D$ by (5.49), because $\bar{B}_{5}$ is convex, and by (4.10). So $\widetilde{\varphi}_{t}(D) \subset D$ in all cases.

The continuity in $t$ of $\widetilde{\varphi}_{t}(y)$ (as in (4.16)) is clear. For the analogue of (4.17), we need to check that $\widetilde{\varphi}_{t}(y)=y$ when $t=0$ and when $y \in$ $\mathbb{R}^{d} \backslash D$. When $t=0$, this follows directly from (4.17). When $t \leq 1 / 2$ and $y \in \mathbb{R}^{d} \backslash D$, this is just (4.17) again. When $t>1 / 2$ and $y \in \mathbb{R}^{d} \backslash D$, we observe that $\varphi_{1}(y)=y$ by (4.17), then $g\left(\varphi_{1}(y)\right)=y$ by (5.45) and (4.10), so $\widetilde{\varphi}_{t}(y)=y$ by $(5.49)$.

For (4.18), we need to check that $\operatorname{dist}\left(\widetilde{\varphi}_{t}(y), E\right)<\alpha r_{1}$ when $y \in$ $E_{\rho} \cap D$ and $0 \leq t \leq 1$. When $t \leq 1 / 2$, this comes from (4.18) for the $\varphi_{t}$. For $t>1 / 2$, first observe that

$$
\varphi_{1}(y) \in V
$$

by (4.19), so $\operatorname{dist}\left(\varphi_{1}(t), E\right) \leq \frac{\alpha r_{1}}{2}+\sqrt{n} L$, by (4.11). Also, (5.47) says that $\left|g\left(\varphi_{1}(y)\right)-\varphi_{1}(y)\right| \leq 4 \sqrt{n} L$. Since by $(5.49) \widetilde{\varphi}_{t}(y)$ is a convex combination of $\varphi_{1}(y)$ and $g\left(\varphi_{1}(y)\right)$, we get that $\operatorname{dist}\left(\widetilde{\varphi}_{t}(t), E\right) \leq \frac{\alpha r_{1}}{2}+$ $5 \sqrt{n} L<\alpha r_{1}$, by (4.6) and as needed for the analogue of (4.18).

We also need to check (4.19), i.e., that $\widetilde{\varphi}_{1}(y) \in V$ when $y \in E_{\rho} \cap$ $D$. This follows from (5.50) and (5.34). So our family $\left\{\widetilde{\varphi}_{t}\right\}$ satisfies (4.14)-(4.19). In addition, $F_{4}=g(F)=g\left(\varphi_{1}\left(E_{\rho} \cap D\right)\right)=\widetilde{\varphi}_{1}\left(E_{\rho} \cap D\right)$ by (5.31), (5.33), (4.14), and (5.49). Since we already know from (5.32) that $F_{4} \in \mathcal{F}_{0}$, we get that $F_{4} \in \mathcal{F}$ (at last!).

Recall that $F$ is a minimizer for $J$. Then $J(F) \leq J\left(F_{4}\right)$. Also recall that $F_{4}=\pi\left(F_{3}\right)$, where $\pi$ was designed to leave untouched all the $d$-dimensional faces of $\mathcal{S}_{d}$, except the faces $T \in \mathcal{A}$ where $F_{3} \cap T$ was sent to $\partial T$. So $F_{4}$ is contained in $F_{3}$, except perhaps for the image by $\pi$ of the $(d-1)$-dimensional part of $F_{3}$ that did not live on $\mathcal{S}_{d}$ (see (5.28)), and a few $(d-1)$-dimensional faces $\partial T$. Thus $J\left(F_{4}\right) \leq J\left(F_{3}\right)$, and altogether

$$
J(F) \leq J\left(F_{3}\right) .
$$

We want to analyze this carefully, so we cut $F$ into the disjoint pieces $F \cap W$ and $F \backslash W$, where $W$ is as in (5.4). Then

$$
J(F)=J(F \cap W)+J(F \backslash W),
$$

where for convenience we still set

$$
J(A)=H^{d}\left(A \cap E_{\rho}\right)+M H^{d}\left(A \backslash E_{\rho}\right)
$$

as in (4.27), even when $A \notin \mathcal{F}$. 
For the next few lines, it will be convenient to set

$$
\Phi=f \circ h \circ \psi \text {. }
$$

Recall that $F_{3}=\Phi(F)$, so $F_{3}=\Phi(F \cap W) \cup \Phi(F \backslash W)$. For $y \in F \backslash W$, $h(\psi(y))=y$ by (5.22). If in addition $y \in F^{*}$ (the finite union of faces that almost cover $F$, see (4.13)), then $y \in \mathcal{S}_{d}$, and (5.24) says that $f(y)=y$. Thus $\Phi(y)=y$ for $y \in F^{*} \backslash W$, and $\Phi\left(F^{*} \backslash W\right)=F^{*} \backslash W$. Since the small set $\Phi\left(F \backslash F^{*}\right)$ does not contribute, $J\left(F_{3}\right) \leq J(\Phi(F \cap W))+J(F \backslash W)$. So (5.51) and (5.52) yield

$$
J(F \cap W) \leq J(\Phi(F \cap W))
$$

and then

$$
H^{d}(F \cap W) \leq M H^{d}(\Phi(F \cap W))
$$

because of the formula (5.53) for $J$.

In view of (5.7), we would prefer to have $\psi(F \cap W)$ instead of $\Phi(F \cap$ $W)$, so we want to show that $h$ and $f$ do not increase the measure of $\psi(F \cap W)$ too much. First, $h$ is $4^{n}$-Lipschitz (see Lemma 5.8), so

$$
H^{d}(h \circ \psi(F \cap W)) \leq 4^{n d} H^{d}(\psi(F \cap W)) .
$$

Recall from (5.21) that $X=h \circ \psi(F \cap W)$, so the left-hand side of (5.57) is $H^{d}(X)$. We cut $X$ into $X_{1}=X \cap \mathcal{S}_{d}$ and $X_{2}=X \backslash \mathcal{S}_{d}$. By $(5.24), f(z)=z$ on $X_{1}$, so $H^{d}\left(f\left(X_{1}\right)\right)=H^{d}\left(X_{1}\right)$.

$$
\begin{aligned}
H^{d}\left(f\left(X_{2}\right)\right) & \leq \sum_{Q \in \mathcal{Q}(\rho)} H^{d}\left(f\left(X_{2} \cap Q\right)\right) \\
& \leq C \sum_{Q \in \mathcal{Q}(\rho)} H^{d}\left(f\left(\bar{X} \cap Q \backslash \mathcal{S}_{d}\right)\right) \\
& \leq C \sum_{Q \in \mathcal{Q}(\rho)} H^{d}\left(\bar{X} \cap Q \backslash \mathcal{S}_{d}\right) \\
& \leq C^{\prime} H^{d}\left(\bar{X} \backslash \mathcal{S}_{d}\right),
\end{aligned}
$$

by (5.27) and because the cubes $Q$ have bounded overlap. Altogether,

$$
\begin{aligned}
H^{d}(\Phi(F \cap W)) & =H^{d}(f(X)) \\
& \leq H^{d}\left(f\left(X_{1}\right)\right)+H^{d}\left(f\left(X_{2}\right)\right) \\
& \leq H^{d}\left(X_{1}\right)+C^{\prime} H^{d}\left(\bar{X} \backslash \mathcal{S}_{d}\right)
\end{aligned}
$$

by (5.54), (5.21), and (5.58). Next we check that

$$
\mathcal{H} \operatorname{dim}\left(\bar{X} \backslash\left[X \cup \mathcal{S}_{d}\right]\right) \leq d-1
$$


Indeed let $z \in \bar{X} \backslash X$ be given. Recall from (5.21) that every point of $X$ is of the form $h(\psi(y))$ for some $y \in F \cap W$. Since $W \Subset B_{2}$ and the domain of definition $V_{+}$of $h$ is closed, we can also write $z=h(\psi(y))$, but with $y \in F \cap \bar{W}$. In addition, $y \notin W$, because $z \notin X$. So $\psi(y)=y$. But $y \in F \subset V$ by (5.17), so $h(y)=y$ by (5.11). Altogether $z=h(\psi(y))=$ $y \in F$. If $z \in F^{*}$, then it lies on $\mathcal{S}_{d}$. So $\bar{X} \backslash\left[X \cup \mathcal{S}_{d}\right] \subset F \backslash F^{*}$, and (5.60) follows from (4.13).

Because of (5.60), the right-hand side of (5.59) is at most $H^{d}\left(X_{1}\right)+$ $C^{\prime} H^{d}\left(X \backslash \mathcal{S}_{d}\right)=H^{d}\left(X_{1}\right)+C^{\prime} H^{d}\left(X_{2}\right) \leq C^{\prime} H^{d}(X)$. Altogether,

$$
\begin{aligned}
H^{d}(F \cap W) & \leq M H^{d}(\Phi(F \cap W)) \\
& \leq C^{\prime} M H^{d}(X) \\
& =C^{\prime} M H^{d}(h \circ \psi(F \cap W)) \\
& \leq 4^{n d} C^{\prime} M H^{d}(\psi(F \cap W))
\end{aligned}
$$

by (5.56), (5.59), (5.21), and (5.57).

Recall from (5.2) that $S=F \cap B_{2}$ and from (5.6) that $W \subset B_{2}$. So $S \cap W=F \cap W$, and (5.61) is just the same as (5.7).

This completes our proof of quasiminimality for $F$. Thus (5.2) holds (with $k=4^{n d} C^{\prime} M$ ), and we shall be able to apply the results of [DS3].

\section{Local uniform rectifiability and flatness}

In this section we use the local uniform rectifiability of $F^{*}$ that comes from (5.2) to show that $F^{*}$ is often flat, derive a contradiction with our assumption (1.2), and thus complete the proof of Lemma 4.1.

Let us first apply the main result of [DS3], which is stated on pp. 10 and 11. Call $S^{*}$ the closed support in $B_{2}$ of the restriction of $H^{d}$ to $S$, as in (1.12) on [DS3, p. 9]. That is,

$$
S^{*}=\left\{x \in B_{2} ; H^{d}(S \cap B(x, r))>0 \text { for all } r>0\right\} .
$$

Recall from (5.2) that $S=F \cap B_{2}$. Since $F$ is a finite union $F^{*}$ of $d$-faces, plus a set of Hausdorff dimension at most $d-1$ (see the definition of $\mathcal{F}_{0}$ below (4.12)), we get that

$$
S^{*}=F^{*} \cap B_{2} \text {. }
$$

Theorem 2.11 in [DS3] says that $S^{*}$ is locally Ahlfors-regular and uniformly rectifiable in $B_{2}$. More precisely, for each $y \in S^{*} \cap B_{1}$, there is a closed, Ahlfors-regular and uniformly rectifiable set $Z_{y}$ of dimension $d$ such that

$$
S^{*} \cap B(y, \delta / 2) \subset Z_{y} \subset S^{*} \cap B(y, \delta) .
$$


Recall from (4.4) that $B_{1}$ is a ball a little bit smaller than $B_{2}$, and from (5.3) and (4.6) that $\delta=4^{-n-1} L \leq \eta r_{1} / 100$. In the statement of [DS3] one allows all balls $B(y, R)$ centered on $S^{*}$, with radius $R<\delta$, and such that $B(y, 3 R) \subset B_{2}$, but we shall only need the ones above.

We should also put stress on the fact that the Ahlfors-regularity and uniform rectifiability constants for the $Z_{y}$ depend on our various constants, but not on $\rho$ or $\alpha_{2}$. The convention in this section will be that $C$ denotes a constant that depends on $n, d, \alpha, \eta, \delta_{1}$, and even $C_{1}$, but in particular not on $\rho$ or $\alpha_{2}$.

Let us not define uniform rectifiability here, and refer to [DS1] for all relevant information, but recall that Ahlfors-regular means that there is a constant $C$ such that

$$
C^{-1} t^{d} \leq H^{d}\left(Z_{y} \cap B(z, t)\right) \leq C t^{d} \text { for } z \in Z_{y} \text { and } 0<t \leq \operatorname{diam}\left(Z_{y}\right) .
$$

In the present case, we also have that

$$
\delta / 4 \leq \operatorname{diam}\left(Z_{y}\right) \leq 2 \delta .
$$

This is Remark 2.15 in [DS3]; grosso modo, the point is that if $\operatorname{diam}\left(S^{*} \cap\right.$ $B(y, R))<\delta / 4$ we could contract $S^{*} \cap B(y, R)$ into a point, and this would contradict the quasiminimality of $S$.

Cover $S \cap B_{1}$ with less than $C$ balls $B(y, \delta / 2)$ centered on $S^{*} \cap B_{1}$, and call $Z$ the corresponding union of sets $Z_{y}$. Then $Z$ is a uniformly rectifiable Ahlfors-regular set, of diameter comparable to $\delta$ and $r_{1}$, and

$$
S^{*} \cap B_{1} \subset Z \text {. }
$$

[For the reader that would be worried about taking (finite) unions of uniformly rectifiable sets, let us say that this is just a matter of convenience, and we could have worked with the various $Z_{y}$ too.]

We shall not work with uniform rectifiability directly, but with one of its consequences, the "weak geometric lemma". Set

$$
\beta_{Z}(y, t)=\inf _{P \in \mathcal{P}}\left\{\sup \left\{t^{-1} \operatorname{dist}(z, P) ; z \in Z \cap B(y, t)\right\}\right\}
$$

for $y \in Z$ and $t>0$, where $\mathcal{P}$ still denotes the set of affine $d$-planes. This is the same thing as $\beta_{E}$ in (1.2), but relative to $Z$. Set

$$
\varepsilon=\frac{\varepsilon_{0}}{10},
$$

where $\varepsilon_{0}$ is still the constant in (1.2), and consider the bad set

$$
\mathcal{B}(\varepsilon)=\left\{(y, t) ; y \in Z, 0<t<\delta, \text { and } \beta_{Z}(y, t)>\varepsilon\right\} .
$$


The weak geometric lemma says that there is a constant $C(\varepsilon)>1$, that depends only on $\varepsilon$ and the Ahlfors-regularity and uniform rectifiability constants for $Z$, such that

$$
\int_{y \in Z \cap B\left(y_{0}, R\right)} \int_{0<t<R} \mathbf{1}_{\mathcal{B}(\varepsilon)} \frac{d H^{d}(y) d t}{t} \leq C(\varepsilon) R^{d}
$$

for $y_{0} \in Z$ and $0<R<\operatorname{diam}(Z)$. The simplest reference for this is probably the following. Theorem 2.4 on [DS1, p. 32] says that uniform rectifiability implies the bilateral weak geometric lemma (BWGL). The BWGL is defined on [DS1, p. 32], and the reader may easily check that it is stronger than the weak geometric lemma (or (6.10)). Let us also say that since [DS1] prefers to talk about unbounded Ahlfors-regular sets $Z$, we may need to apply their Theorem 2.4 to $Z^{\prime}=Z \cup P$, where $P$ is some $d$-plane in $\mathbb{R}^{n}$ such that $\operatorname{dist}\left(P, B_{1}\right) \leq r_{1}$. It is very easy to check that $Z^{\prime}$ is uniformly rectifiable (once you are given the definition), and the weak geometric lemma for $Z^{\prime}$ clearly implies (6.10).

So we have (6.10). It will be convenient to set

$$
d \mu=\frac{d H^{d}(y) d t}{t} .
$$

Note that because of $\frac{d t}{t}, \mu$ is locally infinite (and essentially scale invariant).

Actually, we shall only use (6.10) at one scale. That is, we shall only need to know that

$$
\mu(\mathcal{B}(\varepsilon))=\iint_{(y, t) \in \mathcal{B}(\varepsilon)} \frac{d H^{d}(y) d t}{t} \leq C(\varepsilon) r_{1}^{d},
$$

which is an easy consequence of (6.10). [If you are worried about the measure of the part of $\mathcal{B}(\varepsilon)$ where $\operatorname{diam}(Z)<t<\delta$, note that $\int_{y \in Z} \int_{10^{-2} \delta \leq t \leq \delta} d \mu \leq 10 H^{d}(Z) \leq C r_{1}^{d}$.]

Since $\mu$ is infinite, $(6.12)$ really says that $\mathcal{B}(\varepsilon)$ is small, or equivalently that $\beta_{Z}(y, t) \leq \varepsilon$ most of the time. The general idea now to use this and the fact that

$$
\begin{aligned}
H^{d}\left(Z \cap E_{\rho} \cap B_{0}\right) & \geq H^{d}\left(S^{*} \cap E_{\rho} \cap B_{0}\right) \\
& =H^{d}\left(F^{*} \cap E_{\rho} \cap B_{0}\right) \\
& =H^{d}\left(F \cap E_{\rho} \cap B_{0}\right) \geq \delta_{1} r_{1}^{d} / 2,
\end{aligned}
$$

by $(6.6),(6.2),(4.13)$, and (4.32), to find some place where $E_{\rho}$ is very flat and get a contradiction. Since apparently we cannot use density 
results to find directly large balls where $E_{\rho}$ and $S^{*}$ essentially coincide, we shall have to have resort to a slightly more complicated accounting argument.

Let $C_{2}$ be a large constant, to be chosen soon; $C_{2}$ will depend on many things, including $\varepsilon_{0}$, but this is still all right. Set

$$
\mathcal{G}_{0}=\left\{(y, t) ; y \in Z \cap E_{\rho} \cap B_{0} \text { and } C_{2} \rho \leq t<\delta\right\},
$$

and then

$$
\mathcal{G}=\left\{(y, t) \in \mathcal{G}_{0} ; \beta_{Z}(y, t) \leq \varepsilon\right\} .
$$

To check that $\mathcal{G}$ is reasonably large, first note that

$$
\mu\left(\mathcal{G}_{0}\right)=H^{d}\left(Z \cap E_{\rho} \cap B_{0}\right) \int_{C_{2} \rho}^{\delta} \frac{d t}{t} \geq \frac{\delta_{1} r_{1}^{d}}{2} \log \left(\frac{\delta}{C_{2} \rho}\right),
$$

by (6.13). Later on, we shall take $\alpha_{2}$ so small (in (4.3)) that this will be as big as we want. But for the moment, let us just record that $\mathcal{G}=\mathcal{G}_{0} \backslash \mathcal{B}(\varepsilon)$, so

$$
\mu(\mathcal{G}) \geq \mu\left(\mathcal{G}_{0}\right)-\mu(\mathcal{B}(\varepsilon)) \geq \frac{\delta_{1} r_{1}^{d}}{2} \log \left(\frac{\delta}{C_{2} \rho}\right)-C(\varepsilon) r_{1}^{d},
$$

by $(6.12)$. Next we want to give an upper bound for $\mu(\mathcal{G})$ that will contradict (6.17).

Our plan is to associate a reasonably large piece of $E_{\rho}$ to each pair $(y, t) \in \mathcal{G}$, with reasonable overlap, and then deduce our upper bound from (3.4).

So let $(y, t) \in \mathcal{G}$ be given. Since $\beta_{Z}(y, t) \leq \varepsilon$, we can find a $d$-plane $P$ such that

$$
\operatorname{dist}(z, P) \leq \varepsilon t \text { for } z \in Z \cap B(y, t) .
$$

Besides, $y \in E_{\rho}$ by definition of $\mathcal{G}_{0}$. Thus we can find $\xi \in E$ such that

$$
|\xi-y| \leq \sqrt{n} \rho \leq C_{2} \rho / 10 \leq t / 10,
$$

by (3.2), if $C_{2}$ is large enough, and because $(y, t) \in \mathcal{G}_{0}$. By (1.2), $\beta_{E}(\xi, t / 2) \geq \varepsilon_{0}$, and so we can find $\zeta \in E \cap B(\xi, t / 2)$ such that

$$
\operatorname{dist}(\zeta, P) \geq \frac{\varepsilon_{0} t}{3}=\frac{10 \varepsilon t}{3} \text {. }
$$

Set

$$
D(y, t)=E_{\rho} \cap B(\zeta, \varepsilon t) .
$$

Note that $|\zeta-y| \leq|\xi-y|+t / 2 \leq t / 10+t / 2=6 t / 10$ (by (6.19)), so

$$
D(y, t) \subset E_{\rho} \cap B(y, t) \text {. }
$$


Also, $\operatorname{dist}(\zeta, Z \cap B(y, t)) \geq \operatorname{dist}(\zeta, P)-\varepsilon t \geq \frac{10 \varepsilon t}{3}-\varepsilon \geq 2 \varepsilon t$ by $(6.18)$ and $(6.20)$, and $\operatorname{dist}(\zeta, Z \backslash B(y, t)) \geq 4 t / 10$ because $|\zeta-y| \leq 6 t / 10$. Altogether, $\operatorname{dist}(\zeta, Z) \geq 2 \varepsilon t$ and

$$
\operatorname{dist}(D(y, t), Z) \geq \varepsilon t .
$$

These estimates will help with the bounded overlap. But first we want to show that $D(y, t)$ is not too small. We claim that

$$
H^{d}(D(y, t))=H^{d}\left(E_{\rho} \cap B(\zeta, \varepsilon t)\right) \geq \delta_{1}(\varepsilon t)^{d} .
$$

The first part comes from (6.21). For the second part, let us try to apply TND to the ball $B(\zeta, \varepsilon t)$. First we check (3.3) with $x_{1}=\zeta$ and $r_{1}=\varepsilon t$. Clearly $\zeta \in E$, $\varepsilon t \leq \delta \leq r_{0}$, and $\rho \leq C_{2}^{-1} t \leq(2 \sqrt{n})^{-1} \alpha \varepsilon t$ because $(y, t) \in \mathcal{G}_{0}$ and if $C_{2}$ is large enough. If the analogue of (3.4) does not hold, i.e., if $H^{d}\left(E_{\rho} \cap B(\zeta, \varepsilon t)\right)>C_{1}(\varepsilon t)^{d}$, we are happy because (6.24) is satisfied (with a much better constant). Otherwise, we get a smaller ball $B \subset B(\zeta, \varepsilon t)$ where (3.6) holds for all appropriate families $\left\{\varphi_{t}\right\}$ of deformations. We take $\varphi_{t}(y)=y$ everywhere, which satisfies the required conditions trivially, and now (3.6) says that $H^{d}\left(E_{\rho} \cap B(\zeta, \varepsilon t)\right) \geq$ $H^{d}\left(E_{\rho} \cap B\right) \geq \delta_{1}(\varepsilon t)^{d}$. So (6.24) holds in all cases.

We are now ready for the accounting. In the following computations, we assume that all functions are measurable. One could avoid this difficulty by taking exterior measures, or by discretizing the computations, but we prefer to ignore the issue. First write that

$$
\begin{aligned}
\mu(\mathcal{G})=\iint_{(y, t) \in \mathcal{G}} \frac{d H^{d}(y) d t}{t} & \\
& \leq C \iiint_{(y, t) \in \mathcal{G} ; w \in D(y, t)} \frac{d H^{d}(w) d H^{d}(y) d t}{t^{d+1}}
\end{aligned}
$$

by (6.11) and (6.24), and where we do not write the dependence on $\varepsilon$ either. Next we want to exchange the order of integration. First observe that $w \in E_{\rho} \cap B(y, t) \subset E_{\rho} \cap B(x, r)$ by (6.22), because $y \in B_{0}$ by (6.14), and because $t \leq \delta<\eta r_{1} / 100$ by (6.14), (5.3), and (4.6). Thus

$$
\mu(\mathcal{G}) \leq C \int_{w \in E_{\rho} \cap B(x, r)} I(w) d H^{d}(w)
$$

where the inside integral is

$$
I(w)=\iint_{(y, t) \in \mathcal{G} ; w \in D(y, t)} \frac{d H^{d}(y) d t}{t^{d+1}} .
$$

Now fix $w \in E_{\rho} \cap B(x, r)$ and $t \leq \delta$, and integrate in $y$ first. Note that $y \in Z \cap B(w, t)$, by (6.14) and (6.22). By local Ahlfors-regularity of $Z$ 
(see (6.4)), $H^{d}(Z \cap B(w, t)) \leq C t^{d}$, and when we integrate again in $t$ we get that

$$
I(w) \leq \int_{t \in T(w)} H^{d}(Z \cap B(w, t)) \frac{d t}{t^{d+1}} \leq C \int_{t \in T(w)} \frac{d t}{t},
$$

where now we integrate over the set $T(w)$ of radii $t$ for which we can find $y$ such that $w \in D(y, t)$. Note that if $w \in D(y, t)$, then $\operatorname{dist}(w, Z) \geq$ $\operatorname{dist}(D(y, t), Z) \geq \varepsilon t$ by $(6.23)$, and on the other hand $\operatorname{dist}(w, Z) \leq$ $|w-y| \leq t$. So $T(w) \subset\left[\operatorname{dist}(w, Z), \varepsilon^{-1} \operatorname{dist}(w, Z)\right], I(w) \leq C$, and $(6.26)$ yields

$$
\mu(\mathcal{G}) \leq C H^{d}\left(E_{\rho} \cap B(x, r)\right) \leq C C_{1} r_{1}^{d},
$$

because $B(x, r) \subset B\left(x_{1}, r_{1}\right)$ and we assumed that (4.2) fails.

When we compare this with $(6.17)$, we get that

$$
\frac{\delta_{1} r_{1}^{d}}{2} \log \left(\frac{\delta}{C_{2} \rho}\right)-C(\varepsilon) r_{1}^{d} \leq C C_{1} r_{1}^{d} .
$$

Maybe we should recall here that our constants $\delta_{1}, \delta$ (defined by (5.3) and (4.6)), and $C_{2}$ (see near (6.14)) are now chosen, and that we still have the right to take $\alpha_{2}$ (in the statement of Lemma 4.1) as small as we wish. See the comments before (4.3). Also recall from (4.3) that $\rho \leq \alpha_{2} r_{1}$; if we choose $\alpha_{2}$ small enough, $\rho$ is as small as we want compared to $\delta / C_{2}$, and (6.30) fails.

We finally reached the contradiction that proves Lemma 4.1.

\section{A lower bound on Hausdorff dimension}

In this section we deduce Theorem 3.8 from Lemma 4.1. So we continue with the same closed set $E$ as in the statement of Theorem 3.8. First we want to choose a constant $C_{1}$, so that we can apply the lemma. We give the definition now so that the reader will be convinced that there is no vicious circle, but it will look a little weird.

For each $\rho>0$ and each $y \in \mathbb{R}^{n}$, call $R_{\rho}(y)$ the union of the skeletons $S_{d}(Q)$, where $Q \in \mathcal{Q}(\rho)$ meets $\bar{B}(y, 10 \rho)$. Clearly $H^{d}\left(R_{\rho}(y)\right) \leq A \rho^{d}$ for some geometric constant $A=A(n, d)$; we choose $C_{1}=10 A$.

Now we can apply Lemma 4.1. This gives constants $r_{0}$ and $\alpha_{2}$ such that

$$
H^{d}\left(E_{\rho} \cap B(x, r)\right)>C_{1} r^{d}
$$

for all choices of $x \in E, r \in\left(0, r_{0}\right)$, and $\rho \in\left(0, \alpha_{2} r\right]$. Choose an integer $N$ such that $\frac{1}{2} \alpha_{2} \leq N<\alpha_{2}$; this will make our computations look nicer. 
For $x \in E$ and $r \in\left(0, r_{0}\right)$, set $\rho=N^{-1} r$ and pick a maximal collection $H(x, r)$ of points of $E \cap B(x, 2 r)$, subject to the constraint that

$$
|y-z| \geq 10 \rho \text { for } y, z \in H(x, r), y \neq z .
$$

We want to show that

$$
\sharp H(x, r) \geq 10 N^{d}
$$

Let us check first that

$$
\text { the sets } R_{\rho}(y), y \in H(x, r) \text {, cover } E \cap B(x, r) \text {. }
$$

Let $z \in E \cap B(x, r)$ be given. Then $z \in S_{d}(Q)$ for some $Q \in \mathcal{Q}(\rho)$ that meets $E$. Call $y_{1}$ a point of $E \cap Q$. By maximality of $H(x, r)$, we can find $y \in E$ such that $\left|y-y_{1}\right| \leq 10 \rho$. Then $Q$ is one of the cubes that meet $\bar{B}(y, 10 \rho)$, and $S_{d}(Q) \subset R_{\rho}(y)$. This proves (7.4).

Now

$H^{d}(E \cap B(x, r)) \leq \sum_{H(x, r)} H^{d}\left(R_{\rho}(y)\right) \leq A \rho^{d}(\sharp H(x, r))=A N^{-d} r^{d}(\sharp H(x, r))$,

and so

$$
\sharp H(x, r) \geq A^{-1} N^{d} r^{-d} H^{d}(E \cap B(x, r)) \geq A^{-1} N^{d} C_{1}=10 N^{d},
$$

by $(7.1)$.

The simplest way to estimate the dimension of $E$ will be to use (7.4) to embed a Cantor set $T^{\mathbb{N}^{*}}$ in $E$, where $T=\left\{1,2, \ldots, 10 N^{d}\right\}$ is a set with $10 N^{d}$ elements. Set

$$
\rho_{k}=N^{-k} r_{0} / 2 \text { for } k \geq 0 .
$$

We want to define a point $x_{j} \in E$ for every choice of $j \in T^{k}$ and $k \geq 0$.

We start with any point $x \in E$, which we call $x_{\emptyset}$ to start the induction. Then assume that we already constructed the points $x_{j}, j \in T^{k}$, and let us do this for $j \in T^{k+1}$.

Let $j \in T^{k}$ be given, and apply (7.3) to the point $x_{j}$ and the radius $\rho_{k}$. We get a set $H\left(x_{j}, \rho_{k}\right)$ of points of $E \cap B\left(x_{j}, 2 \rho_{k}\right)$, that lie at mutual distances at least $10 \rho_{k+1}$ from each other. Pick $10 N^{d}$ points in this set, and call them $x_{j, 1}, x_{j, 2}, \ldots, x_{j, 10 N^{d}}$. Here we use the convenient notation $(j, \ell)$ for points of $T^{k+1}$ that start with $j$.

This completes our definition of $x_{j}, j \in T^{k}$, by induction on $k$. Note that in the construction above, $\left|x_{j, \ell}-x_{j}\right| \leq 2 \rho_{k}$. An easy induction gives that

(7.6) $\left|x_{j^{\prime}}-x_{j}\right| \leq 3 \rho_{k}$ when $j \in T^{k}, j^{\prime} \in T^{k^{\prime}}, k^{\prime} \geq k$, and $j^{\prime}$ starts with $j$. 
Similarly, recall that the points $x_{j, \ell}$ lie at distances at least $10 \rho_{k+1}$ from each other. Since the further generations will not modify this by more than $6 \rho_{k+2}<\rho_{k+1}$, by $(7.6)$, we get that

(7.7) $\left|x_{j^{\prime}}-x_{j}\right| \geq 9 \rho_{k}$ when the $k$-th components of $j$ and $j^{\prime}$ differ.

Now we put a distance on $K=T^{\mathbb{N}^{*}}$. If $j$ and $j^{\prime}$ are two distinct sequences in $K$, call $k\left(j, j^{\prime}\right)$ the smallest integer $k$ such that $j_{k} \neq j_{k}^{\prime}$, and then set

$$
\operatorname{dist}\left(j, j^{\prime}\right)=\rho_{k\left(j, j^{\prime}\right)}=N^{-k\left(j, j^{\prime}\right)} r_{0} / 2 .
$$

This defines a distance on $K$, for which $K$ is closed.

By (7.6), we can define $h: K \rightarrow E$ by $h(j)=\lim _{k \rightarrow+\infty} x_{j_{1}, j_{2}, \ldots, j_{k}}$, where we write $j=\left(j_{1}, j_{2}, \ldots, j_{k}, \ldots\right)$. Moreover, if we apply (7.6) with $k=$ $k\left(j, j^{\prime}\right)-1$, we get that $\left|h(j)-h\left(j^{\prime}\right)\right| \leq 3 \rho_{k\left(j, j^{\prime}\right)-1}=3 N \operatorname{dist}\left(j, j^{\prime}\right)$.

Similarly, (7.7) and a small limiting argument say that $\left|h(j)-h\left(j^{\prime}\right)\right| \geq$ $9 \rho_{k\left(j, j^{\prime}\right)}=9 \operatorname{dist}\left(j, j^{\prime}\right)$. Altogether, $h$ is biLipschitz, and

$$
\mathcal{H} \operatorname{dim}(E) \geq \mathcal{H} \operatorname{dim}(K) .
$$

Set $d^{*}=\mathcal{H} \operatorname{dim}(K)$. We claim that

$$
d^{*}=d+\frac{\log 10}{\log N}>1
$$

Indeed it is known that $d^{*}$ is given by the equation

$$
\left(10 N^{d}\right)^{-k}=N^{-k d^{*}},
$$

where the left-hand side gives the measure (for the obvious uniform measure on $K$ ) of a box of the $k$-th generation, while the right-hand side is an equivalent of the $d^{*}$-th power of the diameter of the box. See $[\mathbf{F a}]$ for a proof of (7.11) and similar computations on self-similar sets.

When we take the Logarithm of both sides of (7.11) and divide by $-k$, we get $\log 10+d \log N=d^{*} \log N$, which is equivalent to (7.10). Thus $\mathcal{H} \operatorname{dim}(E) \geq d^{*}$, by $(7.9)$.

The constant $N$, and hence also $d^{*}$, depends only on the various constants in the construction, as promised after the statement of the theorem. This completes our proof.

\section{References}

[Al] F. J. Almgren, JR., Existence and regularity almost everywhere of solutions to elliptic variational problems with constraints, Mem. Amer. Math. Soc. 4(165) (1976), 199 pp. 
[BJ1] C. J. Bishop AND P. W. Jones, Hausdorff dimension and Kleinian groups, Acta Math. 179(1) (1997), 1-39.

[BJ2] C. J. Bishop And P. W. Jones, Wiggly sets and limit sets, Ark. Mat. 35(2) (1997), 201-224.

[Da] G. DAvID, Morceaux de graphes lipschitziens et intégrales singulières sur une surface, Rev. Mat. Iberoamericana 4(1) (1988), $73-114$.

[DS1] G. DAVID AND S. SEMmES, "Analysis of and on uniformly rectifiable sets", Mathematical Surveys and Monographs 38, American Mathematical Society, Providence, RI, 1993.

[DS2] G. DAvid And S. Semmes, Quasiminimal surfaces of codimension 1 and John domains, Pacific J. Math. 183(2) (1998), 213-277.

[DS3] G. DAVID AND S. SEMmES, Uniform rectifiability and quasiminimizing sets of arbitrary codimension, Mem. Amer. Math. Soc. 144(687) (2000), 132 pp.

[Du] J. Dugundu, "Topology", Allyn and Bacon, Inc., Boston, Mass., 1966.

[Fa] K. FALCONER, "Fractal geometry. Mathematical foundations and applications", John Wiley \& Sons, Ltd., Chichester, 1990.

[Se1] S. Semmes, A criterion for the boundedness of singular integrals on hypersurfaces, Trans. Amer. Math. Soc. 311(2) (1989), 501-513.

[Se2] S. SEmmes, Finding structure in sets with little smoothness, in: "Proceedings of the International Congress of Mathematicians", Vol. 1, 2 (Zürich, 1994), Birkhäuser, Basel, 1995, pp. 875-885.

[Yu C. YuE, Dimension and rigidity of quasi-Fuchsian representations, Ann. of Math. (2) 143(2) (1996), 331-355.

\section{Mathématiques}

Bâtiment 425

Université de Paris-sud

91405 Orsay Cedex

France

E-mail address: guy.david@math.u-psud.fr 
